\title{
AN INGHAM TYPE PROOF FOR A TWO-GRID OBSERVABILITY THEOREM*
}

\author{
Paola Loreti $^{1}$ And Michel Mehrenberger ${ }^{2}$
}

\begin{abstract}
Here, we prove the uniform observability of a two-grid method for the semi-discretization of the $1 D$-wave equation for a time $T>2 \sqrt{2}$; this time, if the observation is made in $(-T / 2, T / 2)$, is optimal and this result improves an earlier work of Negreanu and Zuazua [C. R. Acad. Sci. Paris Sér. I 338 (2004) 413-418]. Our proof follows an Ingham type approach.
\end{abstract}

Mathematics Subject Classification. 35L05, 65M55, 93B07

Received July 11, 2005. Revised February 10 and April 26, 2006.

Published online December 21, 2007.

\section{INTRODUCTION}

We consider the $1 D$ wave equation:

$$
\left\{\begin{array}{l}
u_{t t}-u_{x x}=0, \quad 0<x<1, \quad 0<t<T \\
u(t, 0)=0, \quad u(t, 1)=0, \quad 0<t<T, \\
u(0, x)=u^{0}(x), \quad u_{t}(0, x)=u^{1}(x), \quad 0<x<1,
\end{array}\right.
$$

which admits a unique solution $u \in C\left([0, T] ; H_{0}^{1}(0,1)\right) \cap C^{1}\left([0, T] ; L^{2}(0,1)\right)$, for $\left(u^{0}, u^{1}\right) \in H_{0}^{1}(0,1) \times L^{2}(0,1)$. The energy of the solution, given by

$$
E(t)=\frac{1}{2} \int_{0}^{1}\left|u_{t}(t, x)\right|^{2}+\left|u_{x}(t, x)\right|^{2} \mathrm{~d} x,
$$

is conserved, that is, $E(t)=E(0), 0 \leq t \leq T$. It is well known that for $T \geq 2$ we have the observability inequality

$$
E(0) \leq C(T) \int_{0}^{T}\left|u_{x}(t, 1)\right|^{2} \mathrm{~d} t
$$

for each solution $u$ of $(1)$, with a constant $C(T)>0$ independent of the initial data $\left(u^{0}, u^{1}\right)$. This inequality means that the energy of the solution can be estimated by the energy concentrated near the endpoint $x=1$ and it is also equivalent to the boundary controllability of the wave equation (see, e.g., [7]). For the reader's convenience, we recall here the corresponding $1 D$ controllability result, which is equivalent to (2). In fact,

\footnotetext{
Keywords and phrases. Uniform observability, two-grid method, Ingham type theorem.

* We wish to thank the referee for his suggestions and E. Zuazua for all his comments useful in improving the quality and presentation of the present paper.

1 Dipartimento di Metodi e Modelli Matematici per le Scienze Applicate, Sapienza Università di Roma, Via A. Scarpa 16, 00161 Roma, Italy; loreti@dmmm.uniroma1.it

2 IRMA, Université Louis Pasteur, 7 rue René Descartes, 67084 Strasbourg, France; mehrenbe@math.u-strasbg.fr
} 
the observability inequality (2) holds if and only if, for any $\left(y_{0}, y_{1}\right) \in L^{2}(0,1) \times H^{-1}(0,1)$, there exists $v \in$ $L^{2}(0, T)$ such that the solution of the controlled wave equation

$$
\left\{\begin{array}{l}
y_{t t}-y_{x x}=0, \quad 0<x<1, \quad 0<t<T \\
y(t, 0)=0, \quad y(t, 1)=v(t), \quad 0<t<T \\
y(0, x)=y^{0}(x), \quad y_{t}(0, x)=y^{1}(x), \quad 0<x<1
\end{array}\right.
$$

satisfies

$$
y(T, x)=0=\partial_{t} y(T, x)=0, \quad 0<x<1 .
$$

Each solution $u$ of (1) satisfies also the extra regularity property

$$
\int_{0}^{T}\left|u_{x}(t, 1)\right|^{2} \mathrm{~d} t \leq C(T) E(0)
$$

with another constant $C(T)>0$. The latter inequality is often called the direct inequality, whereas the first one is called the observability inequality, as we have said before, and we can also name it inverse inequality (cf. [8] or [9]). The direct inequality is relevant for solving the non-homogeneous boundary problem (3) (see [9]) and guarantees that the controlled solution of (3) satisfies that $y \in C\left([0, T] ; L^{2}(0,1)\right) \cap C^{1}\left([0, T] ; H^{-1}(0,1)\right)$.

Finite difference scheme. Now let us consider the classical finite-difference space semi-discretization of the $1 D$-wave equation, with an odd $N \in \mathbb{N}^{*}$ and $h:=1 /(N+1)$ :

$$
\left\{\begin{array}{lll}
u_{j}^{\prime \prime}=\frac{1}{h^{2}}\left(u_{j+1}-2 u_{j}+u_{j-1}\right), & 0<t<T, & j=1,2, \ldots, N, \\
u_{0}=u_{N+1}=0, & 0<t<T, & \\
u_{j}(0)=u_{j}^{0}, \quad u_{j}^{\prime}(0)=u_{j}^{1}, & & j=0, \ldots, N+1 .
\end{array}\right.
$$

For each initial condition $\left(u_{j}^{0}, u_{j}^{1}\right)_{j=0}^{N+1}$ satisfying the compatibility conditions $u_{0}^{0}=u_{N+1}^{0}=u_{0}^{1}=u_{N+1}^{1}=0$, the system (5) has a unique solution, which is explicitly given by

$$
u_{j}(t)=\sum_{|k|=1}^{N} a_{k} \mathrm{e}^{i \lambda_{k}^{0} t} e_{j}^{|k|}, \quad e_{j}^{|k|}=\sin (j|k| \pi h), \quad \lambda_{k}^{0}=\frac{2}{h} \sin \left(k \frac{\pi h}{2}\right),
$$

where the coefficients $\left(a_{k}\right)_{|k|=1}^{N}$ are uniquely determined by the relations

$$
u_{j}^{0}=\sum_{k=1}^{N}\left(a_{k}+a_{-k}\right) e_{j}^{k}, \quad u_{j}^{1}=\sum_{k=1}^{N} i \lambda_{k}^{0}\left(a_{k}-a_{-k}\right) e_{j}^{k}, \quad j=1, \ldots, N .
$$

The energy of the system is given by

$$
E_{h}^{0}(t)=\frac{h}{2} \sum_{j=0}^{N}\left[\left|u_{j}^{\prime}\right|^{2}+\left|\frac{u_{j+1}(t)-u_{j}(t)}{h}\right|^{2}\right]
$$

because it is a discretization of the continuous energy. It is also constant in time: $E_{h}^{0}(t)=E_{h}^{0}(0), 0<t<T$.

Uniform observability inequality. Now, we look for a semi-discrete version of (2), namely, a uniform observability inequality (or indirect inequality): do we have

$$
E_{h}^{0}(0) \leq C(T) \int_{0}^{T}\left|\frac{u_{N}(t)}{h}\right|^{2} \mathrm{~d} t,
$$


with a constant $C(T)>0$ independent of the initial conditions and of $h$ ? It turns out that the answer is negative, as it was first noticed in [5]. This phenomenon is now well known and it is due to the effect of spurious high frequency numerical solutions, and several methods have been designed and analyzed during the last years, allowing us to avoid the blow-up of the observability constant; see for example [18].

Note that such inequality is also related to the boundary uniform controllability of the solutions, independently of the mesh-size and to whether the controls of the semi-discrete scheme converge to those of the wave equation. This topic has been intensively studied these last years. See [17] for a detailed bibliography. Let us emphasize that the main property and difficulty is that the constant $C(T)$ (which may be different at different places) has to be independent of the mesh-size $h$.

As in the continuous case, we may also look for a direct inequality: do we have

$$
\int_{0}^{T}\left|\frac{u_{N}(t)}{h}\right|^{2} \mathrm{~d} t \leq C(T) E_{h}^{0}(0)
$$

with a constant $C(T)>0$ independent of the initial conditions and of $h$ ? The latter inequality is true (see e.g. [5]), and has been proven by a discrete multiplier approach.

Concerning the uniform observability (8), many remedies have been developed and analyzed these last years. We refer the reader to [17] for a survey of existing methods, and we will mention thereafter only the methods that we will deal with.

The filtering method. One remedy is to filter out the high frequencies, as it was introduced in [5]. More precisely, for $0<\alpha<1$, we can consider the subspace of solutions to (5) or (17) satisfying

$$
a_{k}=0, \quad|k| \geq \alpha N
$$

The two-grid method. We can also recover the uniform observability by taking initial data in a subspace formed by slowly oscillating initial data obtained by interpolation from data given in a coarser grid. It is the so-called two-grid method, the main subject of study in our paper. It has been proposed by Glowinski, Li and Lions [2] (in the context of full finite difference and finite element discretizations in 2D). We suppose that $N \in \mathbb{N}^{*}$ is an odd number. Thus let us consider initial conditions satisfying

$$
u_{2 k+1}^{0}=\frac{u_{2 k}^{0}+u_{2 k+2}^{0}}{2}, \quad u_{2 k+1}^{1}=\frac{u_{2 k}^{1}+u_{2 k+2}^{1}}{2}, \quad k=0, \ldots, \frac{N-1}{2}
$$

By using the relations (10) in the Fourier series (6), we get for $k=0, \ldots, \frac{N-1}{2}$ :

$$
\left\{\begin{aligned}
\left(\lambda_{k}^{0}\right)^{2}\left(a_{k}+a_{-k}\right) & =-\left(\lambda_{N+1-k}^{0}\right)^{2}\left(a_{N+1-k}+a_{-N-1+k}\right), \\
\left(\lambda_{k}^{0}\right)^{3}\left(a_{k}-a_{-k}\right) & =-\left(\lambda_{N+1-k}^{0}\right)^{3}\left(a_{N+1-k}-a_{-N-1+k}\right) .
\end{aligned}\right.
$$

By taking the square of the relations and adding the resulting identities, since $\lambda_{k}^{0} \leq \lambda_{N+1-k}^{0}$ for $k=1, \ldots$, $(N-1) / 2$, we obtain

$$
\left|a_{N+1-k}\right|^{2}+\left|a_{-N-1+k}\right|^{2} \leq\left(\nu_{k}\right)^{4}\left(\left|a_{k}\right|^{2}+\left|a_{-k}\right|^{2}\right), \quad \nu_{k}=\frac{\lambda_{k}^{0}}{\lambda_{N+1-k}^{0}}, \quad k=1, \ldots, \frac{N-1}{2} .
$$

Different methods exist for proving the uniform observability in these classes of initial data; we will deal here with two of them.

Multiplier type approach. The two-grid method has been at first analysed by Negreanu and Zuazua in [15], with a discrete multiplier approach. They proved that (8) holds true for $T>4$, within the class (10). 
Ingham type approach. Another classical way to study the observability is to use an Ingham type approach. It consists in using the Fourier series form of the solution under consideration and to use thereafter a theorem of Ingham or a variant of it.

More precisely, by introducing the Fourier expansion (6) of the solutions and then computing the normal derivative, for the two-grid method, inequality (8) takes the form

$$
C(T) \sum_{k=1}^{N}\left|\lambda_{k}^{0}\right|^{2}\left|a_{k}\right|^{2} \leq \int_{0}^{T}\left|\sum_{|k|=1}^{\frac{N-1}{2}} \frac{e_{N}^{k}}{h}\left(a_{k} \mathrm{e}^{i \lambda_{k}^{0} t}+b_{k} \mathrm{e}^{i \mu_{k}^{0} t}\right)\right|^{2} \mathrm{~d} t, \quad \mu_{|k|}^{0}=\lambda_{N+1-|k|}^{0}, \quad \mu_{-|k|}^{0}=\lambda_{-N-1+|k|}^{0}
$$

with sequences $\left(a_{k}\right),\left(b_{k}\right)$ satisfying $(11)$, where $b_{|k|}:=a_{N+1-|k|}$ and $b_{-|k|}:=a_{-N-1+|k|}$. From (12), we have in particular

$$
\left|b_{k}\right|^{2}+\left|b_{-k}\right|^{2} \leq\left(\nu_{k}\right)^{4}\left(\left|a_{k}\right|^{2}+\left|a_{-k}\right|^{2}\right), \quad \nu_{k}=\tan (k \pi h / 2), \quad k=1, \ldots,(N-1) / 2 .
$$

So, let us recall the original Ingham theorem [6]:

Theorem 1.1. Let $\gamma>0$ and let $\left(\nu_{k}\right)$ be a strictly increasing sequence satisfying the gap assumption

$$
\nu_{k+1}-\nu_{k}>\gamma, \quad \text { for } \quad k \in \mathbb{N} \text {. }
$$

Then for $T>\frac{2 \pi}{\gamma}$, we have

$$
c \sum_{k}\left|a_{k}\right|^{2} \leq \int_{0}^{T}\left|\sum_{k} a_{k} \mathrm{e}^{i \nu_{k}} t\right|^{2} \mathrm{~d} t \leq C \sum_{k}\left|a_{k}\right|^{2},
$$

with constants $c, C>0$ independent of the sequence $\left(a_{k}\right)$.

Remark 1.2. The inequality also holds true under the weaker assumption $\nu_{k+1}-\nu_{k}>\gamma$, for $k \geq k_{0}$, for a given integer $k_{0}(c f .[3])$. The constants may then depend on the first frequencies corresponding to $k=1, \ldots, k_{0}$ for which the gap condition is not guaranteed (see [12] for example).

Difficulties for an Ingham type approach. Asking for an Ingham type proof of uniform observability seems quite natural in the context of the $1 D$ wave equation, where the solution is explicitly given by its Fourier series. It has been applied successfully for some semi-discretizations, like the filtering method [5] or the mixed finite element method [1]. In the case of the two-grid method, the situation is trickier, we have to face an infinite number of eigenvalues which can be arbitrarily close to one another. In particular, we cannot apply Theorem 1.1. The literature in such cases is quite rare (see [10], where a situation of this type is considered, which is however different from our problem).

Looking at the Figure 1, one can see that there is a compensation between the gaps of the sequences $\left(\lambda_{k}^{0}\right)$, $\left(\mu_{k}^{0}\right)$ and the coefficient $\nu_{k}$. Indeed in the regions where the gap of $\left(\mu_{k}\right)$ is small, the coefficient $\nu_{k}$ is also small, and the gap of $\left(\lambda_{k}\right)$ is large, so that the term $a_{k} \mathrm{e}^{i \lambda_{k}^{0} t}$ will dominate the term $b_{k} \mathrm{e}^{i \mu_{k}^{0} t}$. On the other hand, when the coefficient $\nu_{k}$ gets larger, the gap of $\left(\mu_{k}\right)$ also becomes larger.

New Ingham type theorems. In order to face the situation above, we develop some new Ingham type theorems, which take care of the situation just mentioned above. We have the following first result.

Theorem 1.3. Let $N \in \mathbb{N}^{*}, \gamma>0, \alpha>1 / 2$ and $M>0$. Let $\left(\lambda_{k}\right)_{|k|=1}^{N}$ and $\left(\mu_{k}\right)_{|k|=1}^{N}$ be finite sequences such that

$$
\begin{gathered}
\lambda_{k+1}-\lambda_{k}>\gamma, k=1, \ldots, N-1,-2, \ldots,-N, \quad \lambda_{1}-\lambda_{-1}>\gamma, \quad \mu_{N}-\lambda_{N}>\gamma, \quad \lambda_{-N}-\mu_{-N}>\gamma \\
\mu_{k}-\mu_{k+1}>\gamma,|k| \geq N-N^{\alpha}, \quad \mu_{k} \geq \mu_{N-N^{\alpha}}, 1 \leq k \leq N-N^{\alpha}, \quad \mu_{k} \leq \mu_{-N+N^{\alpha}},-1 \geq k \geq-N+N^{\alpha} .
\end{gathered}
$$




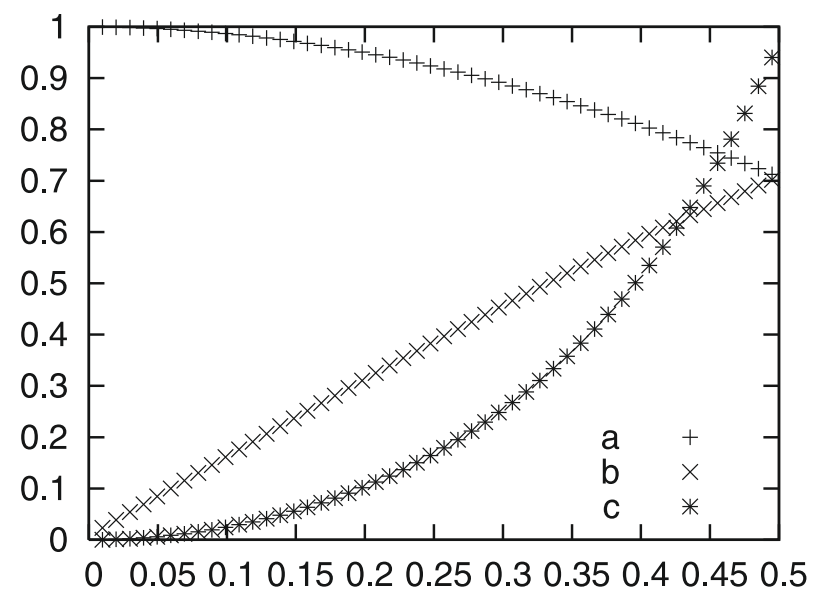

FiguRE 1. Relative gaps $\left(\lambda_{k+1}^{0}-\lambda_{k}^{0}\right) / \pi,\left(\mu_{k}^{0}-\mu_{k+1}^{0}\right) / \pi$ and coefficient $\tan ^{2}(k \pi h / 2)$ vs. $k / N$, for $N=101$ and $k=1, \ldots,(N-1) / 2$; resp. a, b and c on the legend.

Then, for all $T>\frac{2 \pi}{\gamma} \sqrt{\max (1,1 / 2+M)}$, there exists a constant $C(T)>0$ such that

$$
C(T) \sum_{|k|=1}^{N}\left|a_{k}\right|^{2} \leq \int_{0}^{T}\left|\sum_{|k|=1}^{N} a_{k} \mathrm{e}^{i \lambda_{k} t}+b_{k} \mathrm{e}^{i \mu_{k} t}\right|^{2} \mathrm{~d} t
$$

for all sequences of coefficients $\left(a_{k}\right)_{|k|=1}^{N}$ and $\left(b_{k}\right)_{|k|=1}^{N}$ satisfying

$$
\left|b_{k}\right|^{2}+\left|b_{-k}\right|^{2} \leq M^{2}\left(\left|a_{k}\right|^{2}+\left|a_{-k}\right|^{2}\right), \quad k=1, \ldots, N
$$

The new feature in this theorem is that there is no gap assumption for the high frequencies, that are represented by the sequence $\left(\mu_{k}\right)_{|k|=1}^{N-N^{\alpha}}$. We can remark that, for $M>1 / 2$, the lower bound $2 \pi / \gamma(M+1 / 2)$ of the time $T$ is always greater than the bound $2 \pi / \gamma$ corresponding to the first sequence $\left(\lambda_{k}\right)_{|k|=1}^{N}$. In particular, in the application to the two-grid method, the expected bound $2 \sqrt{2}$ of the sequence $\left(\lambda_{k}^{0}\right)_{|k|=1}^{(N-1) / 2}$ cannot be achieved by this theorem. In order to overcome this difficulty, we have developed another generalization of Ingham's theorem, which is the main result of the paper and which will give the sharp time condition $T>2 \sqrt{2}$ for the two-grid method.

Theorem 1.4. Let $N \in \mathbb{N}^{*}$ be an odd number, $h:=\frac{1}{N+1}$ and $f \in C^{3}([-1,1])$ an odd function. Suppose that

- $f^{\prime}(x)>0$ for $0 \leq x<1$;

- $f^{\prime}(1)=0$ and $f^{\prime \prime}(1) \neq 0$.

Define $\gamma>0$ by

$$
\gamma^{2}=\min _{x \in[0,1 / 2]} \min \left(\frac{f^{\prime}(x)^{2}+f^{\prime}(1-x)^{2}}{2}, f^{\prime}(x)^{2}\right),
$$

and set $\lambda_{k}:=\frac{f(k h)}{h}, \gamma_{k}:=\left|f^{\prime}(k h)\right|$ for $|k|=1, \ldots, N$.

Then for each $T>2 \pi / \gamma$, there exists a constant $C(T)>0$ independent of $h$, such that we have

$$
\int_{0}^{T}\left|\sum_{|k|=1}^{N} a_{k} \mathrm{e}^{i \lambda_{k} t}\right|^{2} \geq C(T) \sum_{|k|=1}^{N}\left|a_{k}\right|^{2}
$$


for every sequence $\left(a_{k}\right)$ satisfying

$$
\left|a_{N+1-k}\right|^{2}+\left|a_{-N-1+k}\right|^{2} \leq\left(\frac{\gamma_{N+1-k}}{\gamma_{k}}\right)^{4}\left(\left|a_{k}\right|^{2}+\left|a_{-k}\right|^{2}\right), \quad k=1, \ldots,(N-1) / 2
$$

The main novelty here is that we can mix the gap $\gamma_{|k|}$ of the low frequencies $\lambda_{k}$ with the gap $\gamma_{N+1-|k|}$ of the high ones $\mu_{k}$, as if we had a mean gap $\sqrt{\frac{\gamma_{|k|}^{2}+\gamma_{N+1-|k|}^{2}}{2}}$.

Application for the two-grid method. The main result concerning the application to the two-grid method can be formulated as follows:

Theorem 1.5. Let $I$ be an interval of length $|I|>2 \sqrt{2}$. Let $N$ be an odd number, $h:=\frac{1}{N+1}$.

Then there exists a constant $C_{1}(I)$ independent of $h$, such that

$$
E_{h}^{0}(0) \leq C_{1}(I) \int_{I}\left|\frac{u_{N}(t)}{h}\right|^{2} \mathrm{~d} t
$$

for all the solutions of (5), written in the form (6), with

$$
\left|a_{N+1-k}\right|^{2}+\left|a_{-N-1+k}\right|^{2} \leq \nu_{k}^{4}\left(\left|a_{k}\right|^{2}+\left|a_{-k}\right|^{2}\right),
$$

where $\nu_{k}=\tan (k \pi h / 2)$.

In particular, the solution of the two-grid method satisfies these assumptions $(c f$. (12)), so that we get the uniform observability for $|I|>2 \sqrt{2}$. We will discuss in Section 4 the optimality of this result.

Now, we present the plan of the rest of the paper. Section 2 is devoted to analyze the finite difference scheme by an Ingham type approach. We will at first consider the direct inequality and then prove Theorem 1.5. In Section 3 , we study a more general scheme, namely the $\theta$-scheme (if we take $\theta=0$, we recover the finite difference scheme of Sect. 2). We first study the direct inequality by an Ingham type approach. We then give observability results for the two-grid method: we will apply the multiplier method to obtain a general observability result, and as a relevant example, we will use Theorem 1.3 for the finite element semi-discretization (which corresponds to $\theta=1 / 6)$. In Section 4, we will give necessary conditions for having uniform observability for the schemes under consideration. Finally, in Section 5, we will prove the Ingham type theorems, which have permitted us to obtain Theorem 1.5 and the results in Sections 2 and 3.

Notations. In the sequel, the symbol $a \asymp b$ means that there exist two constants $c_{1}, c_{2}>0$ independent of $h$ and of the numbers $a_{k}, b_{k}$ such that $c_{1} a \leq b \leq c_{2} a$. We will use similarly the notations $\preceq$ and $\succeq$.

\section{Results And PRoOfs For the Finite Difference CASE}

\subsection{The direct inequality}

We have already mentioned that the direct inequality always holds by using discrete multipliers (see Sect. 3 for a proof). We may wonder if we can also obtain this result by using Fourier series. We have the following proposition:

Proposition 2.1. Let $N \in \mathbb{N}^{*}$, and a finite sequence $\left(\lambda_{k}\right)_{k=1}^{N}$. Let $\left(M_{k}\right)_{k=1}^{N}$ be a positive finite sequence such that there exist two constants $M, \gamma>0$ verifying

$$
M_{k} \sum_{j,\left|\lambda_{k}-\lambda_{j}\right|<\gamma} M_{j} \leq M
$$


Then for each $T>0$ there exists a constant $C:=C(T, \gamma, M)>0$ such that

$$
\int_{0}^{T}\left|\sum_{k=1}^{N} M_{k} a_{k} \mathrm{e}^{i \lambda_{k} t}\right|^{2} \mathrm{~d} t \leq C \sum_{k=1}^{N}\left|a_{k}\right|^{2}
$$

for all sequences of coefficients $\left(a_{k}\right)_{|k|=1}^{N}$.

As an application, we can obtain a new proof of (9). Such a proof has its own interest, because it certainly could be applied to many other situations.

Proposition 2.2. Let $N \in \mathbb{N}^{*}$ and $h:=\frac{1}{N+1}$. Then, for each $T>0$, there exists a constant $C_{2}(T)$ independent of $h$, such that

$$
\int_{0}^{T}\left|\frac{u_{N}(t)}{h}\right|^{2} \mathrm{~d} t \leq C_{2}(T) E_{h}^{0}(0)
$$

for all the solutions of (5).

Proof of Proposition 2.1. We will use this time Ingham's second method (see e.g. [8]). We use here that $a \preceq b$, for $a \leq c b$, with a number $c$ depending only on $\gamma, T$ and $M$. We define

$$
H(x)= \begin{cases}\cos \left(\frac{\pi}{\gamma} x\right), & \text { if }|x| \leq \frac{\gamma}{2} \\ 0 & \text { otherwise }\end{cases}
$$

Its Fourier transform is given by

$$
h(t)=\int_{-\infty}^{\infty} H(x) \mathrm{e}^{-i t x} \mathrm{~d} x=\frac{2 \gamma \pi \cos (\gamma t / 2)}{\pi^{2}-t^{2} \gamma^{2}} .
$$

Let $g$ be the Fourier transform of the convolution product $G:=H * H$. There exists an interval $\left.I_{\gamma}=\right]-r_{\gamma}, r_{\gamma}[$ such that $1_{I_{\gamma}} \preceq g$, since $g(0)=4 \gamma^{2} / \pi^{2}>0, g=h^{2}$ is continuous, nonnegative and depends only on $\gamma$. On the other hand, we have $|G| \preceq 1_{-\gamma, \gamma}$, since $H$ is continuous and vanishes outside $]-\gamma / 2, \gamma / 2[$. We then obtain

$$
\begin{aligned}
\int_{I_{\gamma}}\left|\sum_{k=1}^{N} a_{k} \mathrm{e}^{i \lambda_{k} t}\right|^{2} \mathrm{dt} \preceq \sum_{\left|\lambda_{k}-\lambda_{j}\right| \leq \gamma} M_{k} M_{j}\left|a_{k}\right|\left|a_{j}\right| \preceq \sum_{\left|\lambda_{k}-\lambda_{j}\right| \leq \gamma} M_{k} M_{j}\left(\left|a_{k}\right|^{2}+\left|a_{j}\right|^{2}\right) \\
\preceq \sum_{\left|\lambda_{k}-\lambda_{j}\right| \leq \gamma} M_{k} M_{j}\left|a_{k}\right|^{2}=\sum_{k=1}^{N}\left|a_{k}\right|^{2} M_{k} \sum_{j,\left|\lambda_{k}-\lambda_{j}\right| \leq \gamma} M_{j} \preceq \sum_{k=1}^{N}\left|a_{k}\right|^{2},
\end{aligned}
$$

which yields the result since we can replace $I_{\gamma}$ by $[0, T]$ with a classical translation argument.

Proof of Proposition 2.2. The solution is of the form (6) and thus, it suffices to verify condition (15) for the second term, with $M_{k}=|k h| \succeq|\sin (k \pi h)|$. We fix $\gamma=\pi / \sqrt{2}$, such that we have

$$
\left|\mu_{k}-\mu_{j}\right|=\left|\frac{4}{h} \sin \left((k-j) \pi \frac{h}{4}\right) \sin \left((k+j) \pi \frac{h}{4}\right)\right| \succeq\left|k^{2}-j^{2}\right| h,
$$

for $|k|,|j|=1, \ldots, \frac{N-1}{2}$. Thus, the condition (15) may be written as

$$
|k| h^{2} \sum_{j,\left|k^{2}-j^{2}\right|<\delta / h}|j| \preceq 1,
$$


with a constant $\delta>0$. If $k^{2}-\delta / h \geq 0$, we have

$|k| h^{2} \sum_{j,\left|k^{2}-j^{2}\right|<\delta / h}|j| \preceq|k| h^{2} \sqrt{k^{2}+\delta / h}\left(\sqrt{k^{2}+\delta / h}-\sqrt{k^{2}-\delta / h}\right) \preceq|k| h^{2} \delta / h \frac{\sqrt{k^{2}+\delta / h}}{\sqrt{k^{2}+\delta / h}+\sqrt{k^{2}-\delta / h}} \preceq k h \preceq 1$.

On the other hand, if $k^{2}-\delta / h<0$, we obtain

$$
|k| h^{2} \sum_{j,\left|k^{2}-j^{2}\right|<\delta / h}|j| \preceq|k| h^{2} \sum_{j, j^{2} \preceq \delta / h}|j| \preceq|k| h^{2} h^{-1} \preceq 1,
$$

which yields the result.

\subsection{Uniform observability for the two-grid method}

We prove here Theorem 1.5 by applying Theorem 1.4 whose proof is postponed to Section 4 .

Proof of Theorem 1.5. By applying Theorem 1.4 with $f(x)=2 \sin (\pi x / 2)$, we have $\frac{\gamma_{N+1-k}}{\gamma_{k}}=\left|\nu_{k}\right|$, and we get

$$
\int_{0}^{T}\left|\sum_{|k|=1}^{\frac{N-1}{2}} \frac{e_{N}^{k}}{h}\left(a_{k} \mathrm{e}^{i \lambda_{k}^{0} t}+b_{k} \mathrm{e}^{i \mu_{k}^{0} t}\right)\right|^{2} \mathrm{~d} t \geq C(T) \sum_{|k|=1}^{\frac{N-1}{2}}\left|\frac{e_{N}^{k}}{h}\right|^{2}\left(\left|a_{k}\right|^{2}+\left|b_{k}\right|^{2}\right),
$$

for all the sequences $\left(a_{k}\right),\left(b_{k}\right)$ satisfying $\left|b_{k}\right|^{2}+\left|b_{-k}\right|^{2} \leq \nu_{k}^{4}\left(\left|a_{k}\right|^{2}+\left|a_{-k}\right|^{2}\right)$ and for $T>2 \sqrt{2}$. From the last relation, and since $\left|e_{N}^{k} / h\right|=\left|\lambda_{k}^{0} \cos (k \pi h / 2)\right| \geq 1 / 2\left|\lambda_{k}^{0}\right|$, for $k=1, \ldots,(N-1) / 2$, we obtain that

$$
\int_{0}^{T}\left|\sum_{|k|=1}^{\frac{N-1}{2}} \frac{e_{N}^{k}}{h}\left(a_{k} \mathrm{e}^{i \lambda_{k}^{0} t}+b_{k} \mathrm{e}^{i \mu_{k}^{0} t}\right)\right|^{2} \mathrm{~d} t \geq C(T) \sum_{|k|=1}^{\frac{N-1}{2}}\left|\lambda_{k}^{0}\right|^{2}\left|a_{k}\right|^{2} \geq C(T) \sum_{|k|=1}^{N}\left|\lambda_{k}^{0}\right|^{2}\left|a_{k}\right|^{2} .
$$

\section{Results AND PROOFs FOR The $\theta$-SCHEME CASE}

\subsection{Introduction}

We consider here a generalization of the previous scheme (which has been introduced in [13]): the $\theta$-scheme which is obtained by replacing $u_{j}^{\prime \prime}$ with $u_{j}^{\prime \prime}+\theta\left(u_{j+1}^{\prime \prime}-2 u_{j}^{\prime \prime}+u_{j-1}^{\prime \prime}\right)$ in $(5)$ :

$$
\left\{\begin{array}{l}
u_{j}^{\prime \prime}+\theta\left(u_{j+1}^{\prime \prime}-2 u_{j}^{\prime \prime}+u_{j-1}^{\prime \prime}\right)=\frac{1}{h^{2}}\left(u_{j+1}-2 u_{j}+u_{j-1}\right), \quad 0<t<T, \quad j=1,2, \ldots, N \\
u_{0}=u_{N+1}=0, \quad 0<t<T \\
u_{j}(0)=u_{j}^{0}, \quad u_{j}^{\prime}(0)=u_{j}^{1}, \quad j=0, \ldots, N+1
\end{array}\right.
$$

We can notice that (17) is inspired in a dispersive approximation of the wave equation:

$$
u_{t t}-\theta h^{2} \Delta u_{t t}=\Delta u \text {. }
$$

Note that the finite difference scheme corresponds to the case $\theta=0$. The value $\theta=1 / 6$ corresponds to a finite element semi-discretization (see e.g. [14]), and the value $\theta=1 / 4$ can also be derived from a finite element method, by discretizing the position and the velocity differently and enters in the class of Mixed Finite Element methods for approximating a given PDE. 
The solution can be developed in Fourier series as in (6), by replacing $\lambda_{k}^{0}$ with $\lambda_{k}^{\theta}$ which satisfies

$$
-\left(\lambda_{k}^{\theta}\right)^{2}+\theta h^{2}\left(\lambda_{k}^{\theta}\right)^{2}\left(\lambda_{k}^{0}\right)^{2}=-\left(\lambda_{k}^{0}\right)^{2}
$$

More precisely, for each initial condition $\left(u_{j}^{0}, u_{j}^{1}\right)_{j=0}^{N+1}$ satisfying the compatibility conditions $u_{0}^{0}=u_{N+1}^{0}=u_{0}^{1}=$ $u_{N+1}^{1}=0$, system (17) has a unique solution, which is explicitly given by

$$
u_{j}(t)=\sum_{|k|=1}^{N} a_{k} \mathrm{e}^{i \lambda_{k}^{\theta} t} e_{j}^{|k|}, \quad e_{j}^{|k|}=\sin (j|k| \pi h)
$$

where the coefficients $\left(a_{k}\right)_{|k|=1}^{N}$ are uniquely determined by the relations

$$
u_{j}^{0}=\sum_{k=1}^{N}\left(a_{k}+a_{-k}\right) e_{j}^{k}, \quad u_{j}^{1}=\sum_{k=1}^{N} i \lambda_{k}^{\theta}\left(a_{k}-a_{-k}\right) e_{j}^{k}, \quad j=1, \ldots, N .
$$

The energy of the system is given by

$$
E_{h}^{\theta}(t)=\frac{h}{2} \sum_{j=1}^{N}\left(\left|u_{j}^{\prime}\right|^{2}-\theta\left|u_{j+1}^{\prime}-u_{j}^{\prime}\right|^{2}+\left|\frac{u_{j+1}-u_{j}}{h}\right|^{2}\right)
$$

and it satisfies $E_{h}^{\theta}(t)=E_{h}^{\theta}(0)$, for $0<t<T$. Note that $E_{h}^{\theta}(t)$ is positive for $0 \leq \theta \leq 1 / 4$ :

$$
\begin{aligned}
\sum_{j=0}^{N}\left|u_{j}^{\prime}\right|^{2}-\theta\left|u_{j+1}^{\prime}-u_{j}^{\prime}\right|^{2}=\sum_{j=0}^{N}\left|u_{j}^{\prime}\right|^{2}(1-2 \theta) & +2 \theta \sum_{j=0}^{N} u_{j}^{\prime} u_{j+1}^{\prime} \\
\geq & \sum_{j=0}^{N}\left|u_{j}^{\prime}\right|^{2}(1-2 \theta)-\theta\left(\left|u_{j}^{\prime}\right|^{2}+\left|u_{j+1}^{\prime}\right|^{2}\right)=\sum_{j=0}^{N}\left|u_{j}^{\prime}\right|^{2}(1-4 \theta) \geq 0 .
\end{aligned}
$$

The uniform observability or inverse inequality is: do we have

$$
E_{h}^{\theta}(0) \leq C(T)\left(\int_{0}^{T}\left|\frac{u_{N}(t)}{h}\right|^{2} \mathrm{~d} t+\theta \int_{0}^{T}\left|u_{N}^{\prime}(t)\right|^{2} \mathrm{~d} t\right)
$$

with a constant $C(T)>0$ independent of the initial conditions and of $h$ ?

The direct inequality is: do we have

$$
\int_{0}^{T}\left|\frac{u_{N}(t)}{h}\right|^{2} \mathrm{~d} t+\theta \int_{0}^{T}\left|u_{N}^{\prime}(t)\right|^{2} \mathrm{~d} t \leq C(T) E_{h}^{\theta}(0)
$$

with a constant $C(T)>0$ independent of the initial conditions and of $h$ ? 


\subsection{The direct inequality}

The direct inequality has been proven for $\theta=0$ in [5] and [11], for $\theta=1 / 4$ in [1], and for $\theta=1 / 6$ in [14] (and can also be stated from [5]). We shall here give a proof with Fourier series, for $0 \leq \theta \leq 1 / 4$ by applying Proposition 2.1. Note that we could also get the direct inequality with discrete multipliers by establishing the following relation

$$
\int_{0}^{T}\left|\frac{u_{N}}{h}\right|^{2}+\theta\left|u_{N}^{\prime}\right|^{2} \mathrm{~d} t-\left.Y_{h}(t)\right|_{0} ^{T}=2 T E_{h}^{\theta}(0)+(2 \theta-1 / 2) h \int_{0}^{T} \sum_{j=0}^{N}\left(u_{j+1}^{\prime}-u_{j}^{\prime}\right)^{2} \mathrm{~d} t,
$$

with

$$
Y_{h}(t)=h \sum_{j=1}^{N} j\left(u_{j+1}-u_{j-1}\right)\left(u_{j}^{\prime}+\theta\left(u_{j+1}^{\prime}-2 u_{j}^{\prime}+u_{j-1}^{\prime}\right)\right), \quad \text { which satisfies } \quad\left|Y_{h}(t)\right| \leq 2 E_{h}^{\theta}(0) .
$$

Proposition 3.1. For each $0 \leq \theta \leq 1 / 4$ and for $T>0$, there exists a constant $C(T)>0$ such that

$$
\int_{0}^{T}\left|\frac{u_{N}(t)}{h}\right|^{2} \mathrm{~d} t+\int_{0}^{T}\left|u_{N}^{\prime}(t)\right|^{2} \mathrm{~d} t \leq C(T) E_{h}^{\theta}
$$

for all the solutions of (17).

Proof. We have to prove that

$$
\int_{0}^{T}\left|\sum_{k=(N-1) / 2}^{N} \lambda_{k}^{0} a_{k} \mathrm{e}^{i \lambda_{k}^{\theta} t} \frac{e_{N}^{k}}{h}\right|^{2} \leq C(T) \sum_{k=(N-1) / 2}^{N}\left|\lambda_{k}^{0}\right|^{2}\left|a_{k}\right|^{2}
$$

and that

$$
\int_{0}^{T}\left|\sum_{k=(N-1) / 2}^{N} \lambda_{k}^{\theta} e_{N}^{k} a_{k} \mathrm{e}^{i \lambda_{k}^{\theta} t}\right|^{2} \leq C(T) \sum_{k=(N-1) / 2}^{N}\left|\lambda_{k}^{0}\right|^{2}\left|a_{k}\right|^{2}
$$

for $0 \leq \theta<1 / 4$. Thus, we have to check the assumptions of Proposition 2.1, with $M_{k}=|k h|$, since

$$
\left|\frac{e_{N}^{N+1-k}}{h \lambda_{N+1-k}^{0}}\right|=|\sin (k \pi h / 2)| \leq C|k h|, \text { and }\left|\frac{e_{N}^{N+1-k} \lambda_{N+1-k}^{\theta}}{\lambda_{N+1-k}^{0}}\right| \leq C\left|e_{N}^{N+1-k}\right| \leq C|k h| .
$$

By following the proof of Proposition 2.2, we see that we only have to verify that

$$
\left|\lambda_{N+1-k}^{\theta}-\lambda_{N+1-j}^{\theta}\right| \geq C h\left|k^{2}-j^{2}\right|
$$

For this, we set $\varphi(x)=g^{\theta}(1-x)$. Note that $\varphi^{\prime}(x)<0$ for $0<x \leq 1, \varphi^{\prime}(0)=0$ and $\varphi^{\prime \prime}(0) \neq 0$, so that the function $\psi(x)=\varphi(\sqrt{x})$ satisfies $\psi^{\prime}(x) \neq 0$, for $0 \leq x \leq 1$. We then get

$$
\left|\varphi(x)-\varphi\left(x^{\prime}\right)\right| \geq C\left|x^{2}-x^{\prime 2}\right|
$$

which gives (22). 


\subsection{Uniform observability for the two-grid method}

As we will see in Section 4, the uniform observability inequality (19) cannot hold for all initial conditions, for $0 \leq \theta<1 / 4$. It holds however when we apply the two-grid method.

By substituting the relations (10) into the Fourier series (18), we get for $k=0, \ldots, \frac{N-1}{2}$ :

$$
\left\{\begin{array}{l}
\left(\lambda_{k}^{0}\right)^{2}\left(a_{k}+a_{-k}\right)=-\left(\lambda_{N+1-k}^{0}\right)^{2}\left(a_{N+1-k}+a_{-N-1+k}\right) \\
\left(\lambda_{k}^{0}\right)^{2} \lambda_{k}^{\theta}\left(a_{k}-a_{-k}\right)=-\left(\lambda_{N+1-k}^{0}\right)^{2} \lambda_{N+1-k}^{\theta}\left(a_{N+1-k}-a_{-N-1+k}\right) .
\end{array}\right.
$$

By taking the square of the relations and by adding the resulting identities, since $\lambda_{k}^{\theta} \leq \lambda_{N+1-k}^{\theta}$ for $k=$ $1, \ldots,(N-1) / 2$, we obtain

$$
\left|a_{N+1-k}\right|^{2}+\left|a_{-N-1+k}\right|^{2} \leq\left(\nu_{k}\right)^{4}\left(\left|a_{k}\right|^{2}+\left|a_{-k}\right|^{2}\right), \quad \nu_{k}=\frac{\lambda_{k}^{0}}{\lambda_{N+1-k}^{0}}, \quad k=1, \ldots, \frac{N-1}{2} .
$$

The uniform observability has been obtained for the two-grid method [15] (for $\theta=0$ ), [14] (for $\theta=0$ and $\theta=1 / 6)$.

Proposition 3.2. For $0 \leq \theta \leq 1 / 4$, we have

$$
\int_{0}^{T}\left(\left|\frac{u_{N}}{h}\right|^{2}+\theta\left|u_{N}^{\prime}\right|^{2}\right) \mathrm{d} t \geq(T /(1-2 \theta)-4) E_{h}^{\theta}(0)
$$

for all solution of (17) written in the form (18) with (24).

Remark 3.3. • For $\theta=0$, we recover the results $T>4$ obtained in [15] and [14].

- For $\theta=1 / 6$ we get the time $T>2+2 / 3$, which is better than the condition $T>4$ obtained in [14].

- As $\theta$ tends to $1 / 4$, we approach the time 2 of the continuous case. Note that for $\theta=1 / 4$, we do not need the restriction (10) of the initial data: we refer to [1], where the uniform observability of the mixed finite element method is discussed.

- For each $0 \leq \theta \leq 1 / 4$, by applying the same techniques for the filtering method with parameter $\alpha=1 / 2$, we would obtain the same time (see [5], where the case $\theta=0$ and $\theta=1 / 6$ are discussed).

Proof. We set $C_{\theta}:=\frac{1-4 \theta}{1-2 \theta}$, which satisfies for $k=1, \ldots,(N-1) / 2$

$$
(1 / 2-2 \theta)\left|\lambda_{N+1-k}^{\theta}\right|^{2} h^{2} \geq C_{\theta} .
$$

Thus, thanks to the choice of the initial data, we obtain

$(2 \theta-1 / 2) h \sum_{j=0}^{N}\left|u_{j+1}^{\prime}-u_{j}^{\prime}\right|^{2}-C_{\theta} E_{h}^{\theta}=\sum_{|k|=1}^{N}\left((2 \theta-1 / 2) \frac{h^{2}}{2}\left|\lambda_{k}^{0}\right|^{2}\left|\lambda_{k}^{\theta}\right|^{2}-C_{\theta} / 2\left|\lambda_{k}^{0}\right|^{2}\right)\left|a_{k}\right|^{2} \leq \sum_{k=1}^{(N-1) / 2} d_{|k|}\left|\lambda_{k}^{0}\right|^{2} / 2\left|a_{k}\right|^{2}$,

where, for $k=1, \ldots,(N-1) / 2$ and $0 \leq \theta \leq 1 / 4$ we have

$$
\begin{aligned}
& d_{k}:=\left((1 / 2-2 \theta)\left|\lambda_{k}^{\theta}\right|^{2} h^{2}-C_{\theta}\right)+\left.|(1 / 2-2 \theta)| \lambda_{N+1-k}^{\theta}\right|^{2} h^{2}-\left.C_{\theta}|\cdot| \nu_{k}\right|^{2} \\
= & C_{\theta}\left((1 / 2-\theta)\left(\left|\lambda_{k}^{\theta}\right|^{2} h^{2}+\left|\lambda_{N+1-k}^{\theta}\right|^{2} h^{2}\left|\nu_{k}\right|^{2}\right)-1-\left|\nu_{k}\right|^{2}\right)=C_{\theta}\left(\left|\lambda_{N+1-k}^{0}\right|^{2} h^{2}\left(1-\theta h^{2}\left|\lambda_{N+1-k}^{0}\right|^{2}\right)\left(1-\theta h^{2}\left|\lambda_{k}^{0}\right|^{2}\right)\right)^{-1} e_{k},
\end{aligned}
$$

with

$$
\begin{aligned}
e_{k}:= & \left((1 / 2-\theta)\left(\left|\lambda_{k}^{0}\right|\left|\lambda_{N+1-k}^{0}\right|^{2} h^{4}(2-4 \theta)+\left|\nu_{k}\right|^{2}\right)-4\left(1-\theta h^{2}\left|\lambda_{N+1-k}^{0}\right|^{2}\right)\left(1-\theta h^{2}\left|\lambda_{k}^{0}\right|^{2}\right)\right) \\
& =-4+16 \theta+\left|\lambda_{k}^{0}\right|^{2}\left|\lambda_{N+1-k}^{0}\right|^{2} h^{4}\left((-\theta+1 / 2)(2-4 \theta)-4 \theta^{2}\right)=(-1+4 \theta)\left(4-\left|\lambda_{k}^{0}\right|^{2}\left|\lambda_{N+1-k}^{0}\right|^{2} h^{4}\right) \leq 0 .
\end{aligned}
$$




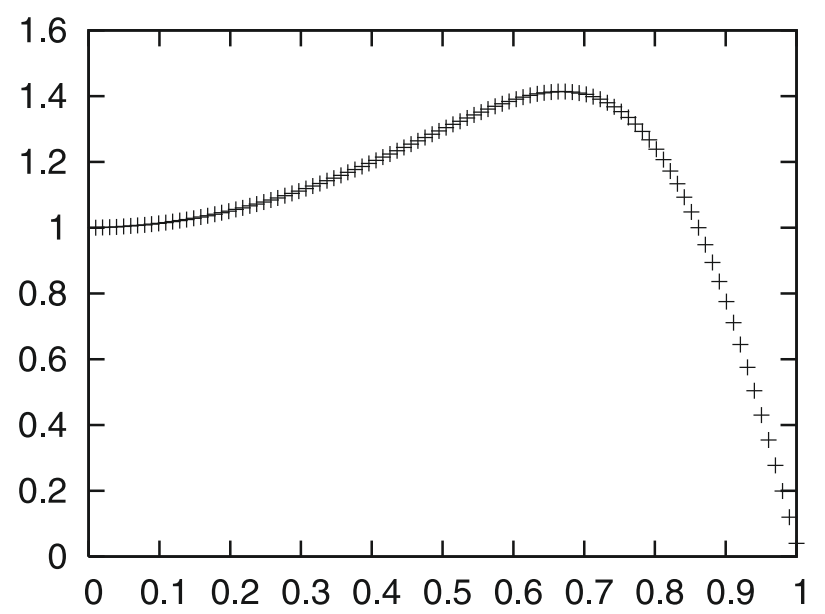

Figure 2. Relative gap $\left(\lambda_{k+1}^{\theta}-\lambda_{k}^{\theta}\right) / \pi v s . k / N$, for $N=101$ and $k=1, \ldots, N$ in the case of the finite element method (i.e. $\theta=1 / 6)$.

We finally get

$$
\int_{0}^{T}\left(\left|\frac{u_{N}}{h}\right|^{2}+\theta\left|u_{N}^{\prime}\right|^{2}\right) \mathrm{d} t \geq\left(\left(2-C_{\theta}\right) T-4\right) E_{h}^{\theta}(0),
$$

which means that $(25)$ holds.

The finite element method $(\theta=1 / 6)$ and the Ingham type approach for the two-grid method. We have already seen in Proposition 3.2 that with the multiplier method, the observability holds for $T>2+2 / 3$. We underline that this holds within the class of two-grid data. By applying an Ingham type approach, we can improve this time as follows. Applying Theorem 1.3 we obtain the observability inequality for

$$
T>2 \pi / \gamma \sqrt{M+1 / 2}=2 \sqrt{3 / 2}=\sqrt{6}(<2.45)
$$

by taking $M=1$ and $\gamma=\pi$. We can improve this further, because the gap $\tilde{\gamma}$ near $k=N / 2$ is larger. We can thus replace $M$ by $M\left(\frac{\gamma}{\tilde{\gamma}}\right)^{2}$. We have here $\gamma=\pi$ and $\tilde{\gamma}=\frac{3 \sqrt{3} \pi}{4}$, so that

$$
M\left(\frac{\gamma}{\tilde{\gamma}}\right)^{2}=16 / 27
$$

and we get the observability property for

$$
T>2 \sqrt{1 / 2+16 / 27}=2 \sqrt{\frac{59}{54}}(<2.1) .
$$

Looking at Figure 2, we see that the gap of the low frequencies (the eigenvalues $\lambda_{k}^{\theta}$, for $|k| \leq(N-1) / 2$ ) is always larger than $\pi$. This implies that the optimal time for the filtered solutions corresponding to the parameter $\alpha=1 / 2$ is 2 (we will recall this fact in the subsection 4 , for the case of the finite difference scheme, but the proof is similar in the finite element method case). We may then obtain 2 as optimal time for the two grid method by using the technic developed in [4]. 


\section{NECESSARY CONDITIONS FOR UNIFORM OBSERVABILITY}

We establish here necessary conditions for the uniform observability, for the different schemes and methods under consideration. Note that such results have already been pointed out (see [5]) and are often explained in terms of the group velocity, by analyzing the dispersion curves (see e.g. [15] and [16]).

Lack of uniform observability for the $\theta$-scheme $(0 \leq \theta<1 / 4)$. Even if the direct inequality (20) holds, it turns out that the inverse inequality (19) fails, as it is explained e.g. in [5] or [17] (there, the inequality (19) was considered for $\theta=0$ and $\theta=1 / 6)$. We will prove here that we cannot have

$$
E_{h}^{\theta} \leq C(T) \int_{0}^{T}\left(\left|\frac{u_{N}(t)}{h}\right|^{2}+\left|u_{N}^{\prime}(t)\right|^{2}\right) \mathrm{d} t
$$

for $0 \leq \theta<1 / 4$.

By substituting (6) into (26), the inequality (26) becomes

$$
C(T) \sum_{|k|=1}^{N}\left|\lambda_{k}^{0}\right|^{2}\left|a_{k}\right|^{2} \leq \int_{0}^{T}\left|\sum_{|k|=1}^{N} a_{k} \mathrm{e}^{i \lambda_{k}^{\theta} t} \frac{e_{N}^{k}}{h}\right|^{2} \mathrm{~d} t+\int_{0}^{T}\left|\sum_{|k|=1}^{N} a_{k} \mathrm{e}^{i \lambda_{k}^{\theta} t} \lambda_{k}^{\theta}\right|^{2} \mathrm{~d} t,
$$

with a constant $C(T)>0$ independent of the sequence $\left(a_{k}\right)_{|k|=1}^{N}$ and of $h$. Since

$$
\int_{0}^{T}\left|a+b \mathrm{e}^{i s t}\right|^{2} \mathrm{~d} t=\int_{0}^{T}\left|a+b+b s \frac{\mathrm{e}^{i s t}-1}{s}\right|^{2} \mathrm{~d} t \leq 2 T|a+b|^{2}+2 s^{2} b^{2} \int_{0}^{T}\left|\frac{\mathrm{e}^{i s t}-1}{s}\right|^{2} \mathrm{~d} t \leq 2 T\left(|a+b|^{2}+s^{2} b^{2}\right),
$$

we would have, by taking $a_{k+1}=1 / \lambda_{k+1}^{0}$ and $a_{k}=-1 / \lambda_{k}^{0}$, and the other coefficients equal to zero in (27):

$C(T) / T \leq|\cos (k \pi h / 2)-\cos ((k+1) \pi h / 2)|^{2}+\left|\lambda_{k}^{\theta} / \lambda_{k}^{0}-\lambda_{k+1}^{\theta} / \lambda_{k+1}^{0}\right|^{2}+\left|\lambda_{k+1}^{s}-\lambda_{k}^{s}\right|^{2}\left(\cos ^{2}(k \pi h / 2)+\left|\lambda_{k}^{\theta} / \lambda_{k}^{0}\right|^{2}\right)$.

Note that $\lambda_{k}^{\theta} / \lambda_{k}^{0}=m(k h)$, with $m(\tau)=(1-4 \theta \sin (\pi \tau / 2))^{-1 / 2}$, which is bounded with bounded derivatives, for $0 \leq \theta<1 / 4$. By taking $k=N-1$, we get

$$
C(T) / T \leq C\left(h^{2}+\left|\lambda_{N}^{\theta}-\lambda_{N-1}^{\theta}\right|^{2}\right) .
$$

Now, we write

$$
\lambda_{k}^{\theta}=\frac{g^{\theta}(k h)}{h}, \quad\left(1-\theta g^{0}(x)^{2}\right) g^{\theta}(x)^{2}=g^{0}(x)^{2}, \quad g^{0}(x)=2 \sin (\pi x / 2) .
$$

We cannot have $g^{\theta}(1)=0$, and thus, since $\left(g^{0}\right)^{\prime}(1)=0$ we get

$$
2 g^{\theta}(1)\left(g^{\theta}\right)^{\prime}(1)\left(1-\theta g^{\theta}(1)^{2}\right)=0 \Rightarrow \theta=\frac{1}{g^{0}(1)^{2}}=\frac{1}{4} \quad \text { or } \quad\left(g^{\theta}\right)^{\prime}(1)=0,
$$

so that we obtain, for $0 \leq \theta<1 / 4: C(T) / T \leq C^{\prime} h^{2}$, since $\left(g^{\theta}\right)^{\prime \prime}$ is bounded. Thus, the uniform observability inequality does not hold. In fact combining more and more frequencies, it can be shown that the constant blows up at an arbitrary large polynomial rate.

The filtering method for the finite difference scheme $(\theta=0)$. By the multiplier method, we can obtain the estimation $T>2 / \cos ^{2}(\alpha \pi / 2)$ for the observability property, whereas we have the finer estimate $T>$ $2 / \cos (\alpha \pi / 2)$ by a Ingham type approach (see [5] for a proof). 
Let us show here that the latter estimation is optimal. Thus, we suppose that the uniform observability holds for a time $T$. We then have

$$
C_{1}(T) \sum_{k}\left|a_{k}\right|^{2} \leq \int_{0}^{T}\left|\sum_{k} a_{k} \mathrm{e}^{i \lambda_{k}^{0} t}\right|^{2} \mathrm{~d} t \leq C_{2}(T) \sum\left|a_{k}\right|^{2}
$$

for each finite sequence $\left(a_{k}\right)$, such that $a_{k}=0$, if $|k h|>\alpha$. In particular, for each $0<\alpha^{\prime}<\alpha$, for $N$ large enough, the latter inequality will hold, for each sequence $a_{k}=0$ such that $\left|k-\alpha^{\prime} / h\right|>N^{1 / 4}$.

If $\left|k-\alpha^{\prime} / h\right| \leq N^{1 / 4}$, by developing $\lambda_{k}^{0}=2 / h \sin \left(\alpha^{\prime} \pi / 2+\left(k-\alpha^{\prime} / h\right) \pi h / 2\right)$, we have

$$
\lambda_{k}^{0}=2 / h \sin \left(\alpha^{\prime} \pi / 2\right)+\pi \cos \left(\alpha^{\prime} \pi / 2\right)\left(k-\alpha^{\prime} / h\right)-\varepsilon_{k}, \quad\left|\varepsilon_{k}\right| \leq 2 / h\left|k-\alpha^{\prime} / h\right|^{2}|\pi h / 2|^{2} \leq \pi^{2} / 2 \sqrt{h} .
$$

Let $\left(a_{k}\right)$ be a sequence whose coefficients are zero except a finite number of them. We can choose $N$ large enough so that, if $|k|>N^{1 / 4}-1$, we have $a_{k}=0$. It follows that

$$
\int_{0}^{T}\left|\sum_{k} a_{k} \mathrm{e}^{i \pi \cos \left(\alpha^{\prime} \pi / 2\right) k t}\right|^{2} \mathrm{~d} t=\int_{0}^{T}\left|\sum_{k} b_{k} \mathrm{e}^{i\left(\lambda_{k}^{0}+\varepsilon_{k}\right) t}\right|^{2} \mathrm{~d} t \geq \int_{0}^{T}\left|\sum_{k} b_{k} \mathrm{e}^{i \lambda_{k}^{0} t}\right|^{2} \mathrm{~d} t-A \geq C(T) \sum_{k}\left|a_{k}\right|^{2}-A
$$

with $b_{k}:=a_{k-\alpha^{\prime} / h}$ (so that if $\left|k-\alpha^{\prime} / h\right|>N^{1 / 4}$, we have $\left.b_{k}=0\right)$, and with

$$
\begin{array}{r}
A:=\int_{0}^{T}\left|\sum_{k} b_{k} \mathrm{e}^{i \lambda_{k}^{0} t}\left(\mathrm{e}^{i \varepsilon_{k} t}-1\right)\right|^{2} \mathrm{~d} t=\int_{0}^{T}\left|\int_{0}^{t} \sum_{k} \varepsilon_{k} b_{k} \mathrm{e}^{i \lambda_{k}^{0} t} \mathrm{e}^{i \varepsilon_{k} s} \mathrm{~d} s\right|^{2} \mathrm{~d} t \leq \int_{0}^{T} t^{2} \int_{0}^{t}\left|\sum_{k} \varepsilon_{k} b_{k} \mathrm{e}^{i \lambda_{k}^{0} t} \mathrm{e}^{i \varepsilon_{k} s}\right|^{2} \mathrm{~d} s \mathrm{~d} t \\
\leq T^{2} \int_{0}^{T} \int_{0}^{T}\left|\sum_{k} \varepsilon_{k} b_{k} \mathrm{e}^{i \lambda_{k}^{0} t} \mathrm{e}^{i \varepsilon_{k} s}\right|^{2} \mathrm{~d} t \mathrm{~d} s \leq T^{3} \pi^{2} / 2 \sqrt{h} \sum_{k}\left|a_{k}\right|^{2},
\end{array}
$$

so that the sequence $\left(\pi \cos \left(\alpha^{\prime} \pi / 2\right) k\right)_{k \in \mathbb{Z}}$ satisfies

$$
\int_{0}^{T}\left|\sum_{k} a_{k} \mathrm{e}^{i \pi \cos \left(\alpha^{\prime} \pi / 2\right) k t}\right|^{2} \mathrm{~d} t \geq C(T) \sum_{k}\left|a_{k}\right|^{2}
$$

and the constant being independent of the sequence $\left(a_{k}\right)$, this inequality cannot hold for $T<2 / \cos \left(\alpha^{\prime} \pi / 2\right)$, and thus also for $T<2 / \cos (\alpha \pi / 2)$, which ends the proof of the optimality.

The two-grid method for the finite difference scheme: optimal time invariant by translation. We have seen in Theorem 1.5 that the uniform observability holds for $|I|>2 \sqrt{2}$ (which is better than the time 4 obtained by the multiplier method), within the class of two-grid data. Note that L. Ignat, in his thesis [4], shows that observability holds in the class of filtered data for all time intervals of length greater than $2 \sqrt{2}$, by using other methods. At this time, we may wonder if this result is optimal. Thus, let us suppose that a uniform observability inequality

$$
E_{h}^{0}(0) \leq C(T) \int_{I}\left|\frac{u_{N}(t)}{h}\right|^{2} \mathrm{~d} t
$$

holds for solutions of the two-grid method, and for every interval $I$ of length greater than $T_{0}$. We shall prove that $T_{0}$ cannot be smaller that $2 \sqrt{2}$, which gives the optimality of the time invariant by translation for the two-grid method. For this, we consider initial data such that

$$
a_{k}=a_{-k}, \quad N^{-}:=N / 2-N^{1 / 4} \leq k \leq N / 2, \quad a_{k}=0 \quad|k|<N^{-}
$$


and suppose that we have (28), for $I=(-T / 2, T / 2)$. We then have

$$
\int_{I}\left|\frac{u_{N}(t)}{h}\right|^{2} \mathrm{~d} t \leq 2 \int_{-T / 2}^{T / 2}\left|\sum_{k \geq N^{-}}^{N / 2} \frac{e_{N}^{k}}{h}\left(a_{k} \mathrm{e}^{i \lambda_{k} t}+a_{k} \nu_{k}^{2} \mathrm{e}^{i \lambda_{N+1-k} t}\right)\right|^{2}+\left|\sum_{k \geq N^{-}}^{N / 2} \frac{e_{N}^{k}}{h}\left(a_{k} \mathrm{e}^{-i \lambda_{k} t}+a_{k} \nu_{k}^{2} \mathrm{e}^{-i \lambda_{N+1-k} t}\right)\right|^{2} \mathrm{~d} t
$$

and thus

$$
C(T) \sum_{k=N^{-}}^{N / 2}\left|\lambda_{k}\right|^{2}\left|a_{k}\right|^{2} \leq \int_{-T / 2}^{T / 2}\left|\sum_{k \geq N^{-}}^{N / 2} \frac{e_{N}^{k}}{h} a_{k} \mathrm{e}^{i \lambda_{k} t}\right|^{2} \mathrm{~d} t+\int_{-T / 2}^{T / 2}\left|\sum_{k \geq N^{-}}^{N / 2} \nu_{k}^{2} \frac{e_{N}^{k}}{h} a_{k} \mathrm{e}^{i \lambda_{N+1-k} t}\right|^{2} \mathrm{~d} t
$$

which gives (by using the fact that $\left|\frac{e_{N}^{k}}{\lambda_{k} h}\right| \geq \cos (\pi / 4)$, as soon as $N / 2-\sqrt{N}-1 \leq k \leq N / 2$ ),

$$
C(T) \sum_{k=N^{-}}^{N / 2}\left|a_{k}\right|^{2} \leq \int_{-T / 2}^{T / 2}\left|\sum_{k \geq N^{-}}^{N / 2} a_{k} \mathrm{e}^{i \lambda_{k} t}\right|^{2} \mathrm{~d} t+\int_{-T / 2}^{T / 2}\left|\sum_{k \geq N^{-}}^{N / 2} \nu_{k}^{2} a_{k} \mathrm{e}^{i \lambda_{N+1-k} t}\right|^{2} \mathrm{~d} t .
$$

By decomposing $\lambda_{k}^{0}=2 / h \sin (\pi / 4+(k-1 /(2 h)) \pi h / 2)$ and $\lambda_{N+1-k}^{0}=2 / h \cos (\pi / 4+(k-1 /(2 h)) \pi h / 2)$, we follow the previous proof and we get

$$
\int_{-T / 2}^{T / 2}\left|\sum_{k} a_{k} \mathrm{e}^{i \pi / \sqrt{2} k t}\right|^{2} \mathrm{~d} t+\int_{-T / 2}^{T / 2}\left|\sum_{k} \nu_{k}^{2} a_{k} \mathrm{e}^{-i \pi / \sqrt{2} k t}\right|^{2} \mathrm{~d} t \geq C(T) \sum_{k}\left|a_{k}\right|^{2} .
$$

Now, by taking an upper bound of $\left|\nu_{k}^{2}-1\right|$ tending to zero, and since the interval is symmetric, this inequality reduces to

$$
\int_{-T / 2}^{T / 2}\left|\sum_{k} a_{k} \mathrm{e}^{i \pi / \sqrt{2} k t}\right|^{2} \mathrm{~d} t \geq C(T) \sum_{k}\left|a_{k}\right|^{2}
$$

and thus we have $T \geq 2 \sqrt{2}$, so that $T_{0}$ can effectively not be smaller than $2 \sqrt{2}$.

An example where observing on $(0, T)$ or on $(-T / 2, T / 2)$ can change. We now look at a simplified case, in order to show that in general it is different to study the observability problem in $(0, T)$ or $\left(-\frac{T}{2}, \frac{T}{2}\right)$.

Let $\alpha>0$. Then we have $T \geq \frac{\pi}{\alpha}$ if and only if

$$
C(T) \sum_{k \geq 1}\left|a_{k}\right|^{2} \leq \int_{0}^{T}\left|\sum_{k \geq 1} a_{k}\left(\mathrm{e}^{i k \alpha t}+\mathrm{e}^{-i k \alpha t}\right)\right|^{2} \mathrm{~d} t
$$

holds for all $\left(a_{k}\right)$. Indeed, we have for $T \geq \pi / \alpha$

$$
\begin{aligned}
& 2 \pi \sum_{k \geq 1}\left|a_{k}\right|^{2}=2 \sum_{k \geq 1} a_{k} \overline{a_{k^{\prime}}} \int_{0}^{\frac{\pi}{\alpha}} \cos \left(\left(k-k^{\prime}\right) \alpha t\right) \mathrm{d} t= \\
& \qquad \int_{0}^{\pi / \alpha}\left|\sum_{k \geq 1} a_{k}\left(\mathrm{e}^{i k \alpha t}+\mathrm{e}^{-i k \alpha t}\right)\right|^{2} \mathrm{~d} t \leq \int_{0}^{T}\left|\sum_{k \geq 1} a_{k}\left(\mathrm{e}^{i k \alpha t}+\mathrm{e}^{-i k \alpha t}\right)\right|^{2} \mathrm{~d} t
\end{aligned}
$$



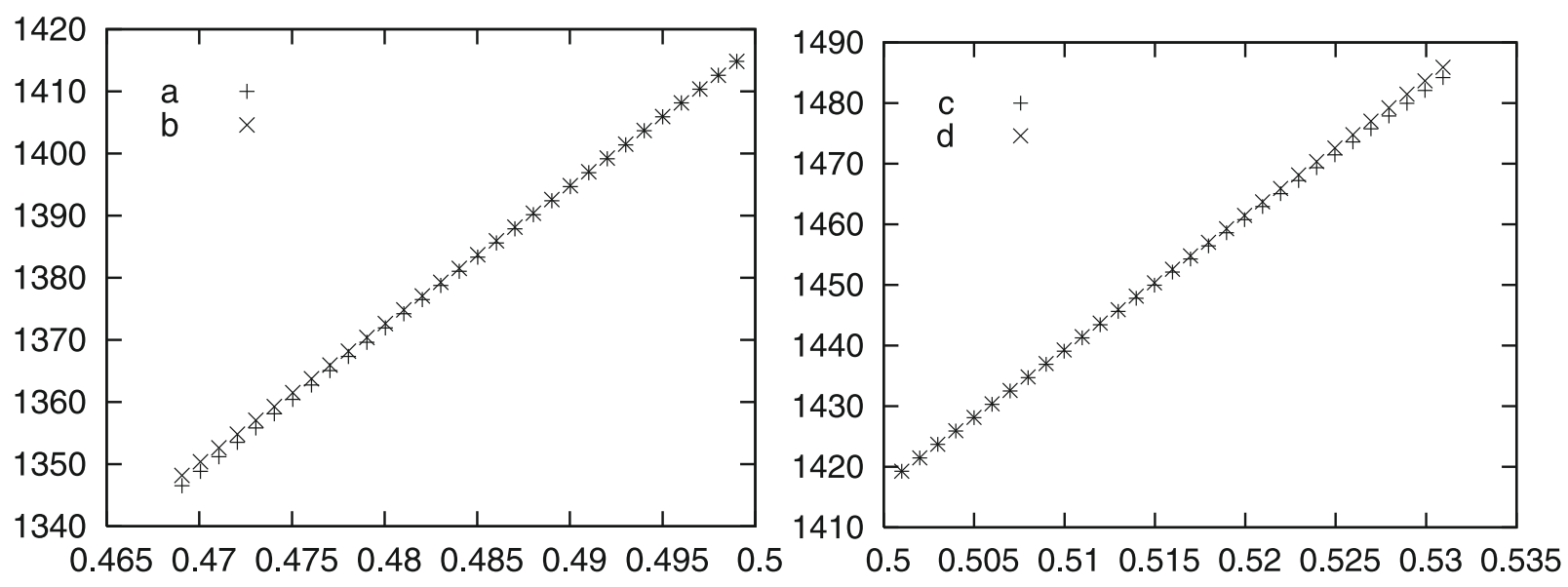

Figure 3. (a) $\lambda_{k}^{0}$, (b) $\lambda_{(N+1) / 2}^{0}+(k-(N+1) / 2) \alpha$, (c) $\lambda_{N+1-k}^{0}$ and (d) $\lambda_{(N+1) / 2}^{0}+((N+$ $1) / 2-k) \alpha$, for $\alpha=\pi / \sqrt{2}, N=1001$ and $k=(N+1) / 2-\sqrt{N}, \ldots,(N-1) / 2$.

and the inequality

$$
C(T) \sum_{k \geq 1}\left|a_{k}\right|^{2} \leq \int_{0}^{T}\left|\sum_{k \geq 1} a_{k}\left(\mathrm{e}^{i k \alpha t}+\mathrm{e}^{-i k \alpha t}\right)\right|^{2} \mathrm{~d} t=\frac{1}{2} \int_{-T}^{T}\left|\sum_{k \geq 1} a_{k}\left(\mathrm{e}^{i k \alpha t}+\mathrm{e}^{-i k \alpha t}\right)\right|^{2} \mathrm{~d} t \leq 2 \int_{-T}^{T}\left|\sum_{k \geq 1} a_{k} \mathrm{e}^{i k \alpha t}\right|^{2} \mathrm{~d} t
$$

can only hold for all $\left(a_{k}\right)$ if $2 T \geq \frac{2 \pi}{\alpha}$. On the other hand, we have $T \geq \frac{2 \pi}{\alpha}$ if and only if

$$
C(T) \sum_{k \geq 1}\left|a_{k}\right|^{2} \leq \int_{-T / 2}^{T / 2}\left|\sum_{k \geq 1} a_{k}\left(\mathrm{e}^{i k \alpha t}+\mathrm{e}^{-i k \alpha t}\right)\right|^{2} \mathrm{~d} t
$$

holds for all $\left(a_{k}\right)$.

Now, we consider again the case of the two-grid method for the finite difference scheme. Note that the behaviour may be similar to the simplified sequence. As an example, Figure 3 illustrates the fact that the sequence $\lambda_{k}^{0}\left(\right.$ resp. $\left.\lambda_{N+1-k}^{0}\right)$ is close to the simplified sequence $\lambda_{(N+1) / 2}^{0}+(k-(N+1) / 2) \alpha\left(\right.$ resp. $\lambda_{(N+1) / 2}^{0}+$ $((N+1) / 2-k) \alpha)$, for $k$ near and smaller than $(N-1) / 2$, with $\alpha=\pi / \sqrt{2}$, for which we have seen that the time of observability can differ if the observation is made on $(0, T)$ or on $(-T / 2, T / 2)$.

Motivated by the latter observation, we state the following question, as an open problem: changing the interval of observation to $(0, T)$, is it possible to observe the system in a shorter time than the optimal time invariant by translation?

Thus, in the case of the two-grid method (i.e. for solutions satisfying only the assumption (23)), the optimal time on $(0, T)$ needs a more careful investigation, whereas the estimate $T>2 \sqrt{2}$ is optimal on $(-T / 2, T / 2)$, as we have seen it in the preceding paragraph. 


\section{Proof of the Ingham type theorems}

In this section we will prove Theorems 1.3 and 1.4 .

Proof of Theorem 1.3. We use Ingham's first method, following at first [10]. We consider the function

$$
G(t)= \begin{cases}\cos \frac{\pi t}{T} & \text { if }|t| \leq T / 2 \\ 0 & \text { si }|t|>T / 2\end{cases}
$$

Its Fourier transform $\tilde{K}$ satisfies

$$
\tilde{K}(\tau)=\int_{-\infty}^{\infty} G(t) \mathrm{e}^{i \tau t} \mathrm{~d} t=-\frac{2 T \pi \cos (\tau T / 2)}{\tau^{2} T^{2}-\pi^{2}}=\frac{2 T}{\pi} K_{T}(\tau)
$$

with

Thus we have

$$
K(\tau)=\frac{\cos \left(\frac{\pi}{2} \tau\right)}{1-\tau^{2}}, \quad K_{T}(\tau)=K\left(\frac{T}{\pi} \tau\right)
$$

$$
\begin{array}{r}
\int_{-T / 2}^{T / 2}\left|\sum_{|k|=1}^{N} a_{k} \mathrm{e}^{i \lambda_{k} t}+b_{k} \mathrm{e}^{i \mu_{k} t}\right|^{2} \mathrm{~d} t \geq \frac{2 T}{\pi} \sum_{|k|,|j|=1}^{N} K_{T}\left(\lambda_{k}-\lambda_{j}\right) a_{k} \overline{a_{j}} \\
\quad+b_{k} \overline{b_{j}} K_{T}\left(\mu_{k}-\mu_{j}\right)+a_{k} \overline{b_{j}} K_{T}\left(\lambda_{k}-\mu_{j}\right)+\overline{a_{k}} b_{j} K_{T}\left(\mu_{k}-\lambda_{j}\right),
\end{array}
$$

because $0 \leq k \leq 1_{[-T / 2, T / 2]}$. On the other hand, since $G$ is positive, we also have

$$
\sum_{|k|,|j|=1}^{N} b_{k} \overline{b_{j}} K_{T}\left(\mu_{k}-\mu_{j}\right)=\int G(t)\left|\sum_{|k|=1}^{N} b_{k} \mathrm{e}^{i \mu_{k} t}\right|^{2} \mathrm{~d} t \geq 0
$$

We thus get rid of these terms, as it was noticed in [10]; note that this argument is not valid if we use Ingham's second method (and thus we have not been able to follow the shorter proof of the result of [10] in [8]). We thus obtain, thanks to the triangle inequality

$$
\begin{aligned}
\int_{-T / 2}^{T / 2}\left|\sum_{|k|=1}^{N} a_{k} \mathrm{e}^{i \lambda_{k} t}+b_{k} \mathrm{e}^{i \mu_{k} t}\right|^{2} \mathrm{~d} t \geq \frac{2 T}{\pi} & {\left[\sum_{|k|=1}^{N} K_{T}(0)\left|a_{k}\right|^{2}\right.} \\
& \left.-\sum_{|k|=1}^{N} \sum_{j \neq k}\left|a_{k}\right|\left|a_{j}\right|\left|K_{T}\left(\lambda_{k}-\lambda_{j}\right)\right|-2 \sum_{|k|,|j|=1}^{N}\left|a_{k}\right|\left|b_{j}\right|\left|K_{T}\left(\lambda_{k}-\mu_{j}\right)\right|\right] .
\end{aligned}
$$

We now have to proceed differently than in [10], in order to treat the coefficients $b_{k}$ whose sum may not be bounded independently of $N$.

We already have from the gap assumption of the sequence $\left(\lambda_{k}\right)$ that $\left|\lambda_{k}-\lambda_{j}\right| \geq \gamma|k-j|$. It follows from the gap assumption on the mixed terms that

$$
\left|\lambda_{k}-\mu_{j}\right|=\mu_{j}-\mu_{N}+\mu_{N}-\lambda_{N}+\lambda_{N}-\lambda_{k} \geq(N-j) \gamma+\gamma+(N-k) \gamma=(2 N+1-j-k) \gamma,
$$

if $k, j \geq N-N^{\alpha}$, and

$$
\left|\lambda_{k}-\mu_{j}\right|=\lambda_{k}-\lambda_{-N}+\lambda_{-N}-\mu_{-N}+\mu_{-N}-\mu_{j} \geq(N+k) \gamma+\gamma+(N+j) \gamma=(2 N+1+j+k) \gamma
$$


if $k, j \leq N^{\alpha}-N$, by using the assumptions

$$
\begin{aligned}
\mu_{k} \leq \mu_{N^{\alpha}-N} \leq \cdots \leq \mu_{-N} \leq \lambda_{-N} \leq \cdots \leq \lambda_{N^{\alpha}-N} & \leq \cdots \leq \lambda_{-1} \\
& \leq \lambda_{1} \leq \cdots \leq \lambda_{N-N^{\alpha}} \leq \cdots \leq \lambda_{N} \leq \mu_{N} \leq \cdots \leq \mu_{N-N^{\alpha}} \leq \mu_{\ell}
\end{aligned}
$$

for $1 \leq \ell \leq N-N^{\alpha}$ and $-1 \geq k \geq N^{\alpha}-N$. We also get

$$
\begin{aligned}
& \left|\lambda_{k}-\mu_{j}\right|=\mu_{j}-\lambda_{k} \geq \mu_{N}-\lambda_{N-N^{\alpha}} \geq \lambda_{N}-\lambda_{N-N^{\alpha}} \geq C N^{\alpha}, \text { if } 1 \leq k \leq N-N^{\alpha}, j \geq 1, \\
& \left|\lambda_{k}-\mu_{j}\right|=\mu_{j}-\lambda_{k} \geq \mu_{N-N^{\alpha}}-\lambda_{N} \geq \mu_{N-N^{\alpha}}-\mu_{N} \geq C N^{\alpha}, \text { if } k \geq 1, j \leq N-N^{\alpha}, \\
& \left|\lambda_{k}-\mu_{j}\right|=\lambda_{k}-\mu_{j} \geq \lambda_{N^{\alpha}-N}-\mu_{-N} \geq \lambda_{N^{\alpha}-N}-\lambda_{-N} \geq C N^{\alpha}, \text { if } N^{\alpha}-N \leq k \leq-1, j \leq-1, \\
& \left|\lambda_{k}-\mu_{j}\right|=\lambda_{k}-\mu_{j} \geq \lambda_{-N}-\mu_{N^{\alpha}-N} \geq \mu_{-N}-\mu_{N^{\alpha}-N} \geq C N^{\alpha}, \text { if } k \leq-1, N^{\alpha}-N \leq j \leq-1 .
\end{aligned}
$$

We thus have $\left|\lambda_{k}-\mu_{j}\right| \geq \gamma d_{k, j}$ with a sequence $d_{k, j}$ satisfying

$d_{k, j}=2 N+1-k-j, \quad k, j \geq N-N^{\alpha}, \quad d_{k, j}=2 N+1-k-j, \quad k, j \leq-N+N^{\alpha}, \quad d_{k, j} \geq C N^{\alpha}, \quad$ otherwise.

We then have

$$
\left|K_{T}\left(\lambda_{k}-\mu_{j}\right)\right| \leq\left(\frac{2 \pi}{T \gamma}\right)^{2} \frac{1}{4 d_{k, j}^{2}-1}, \quad d_{k, j}=d_{j, k}, \quad|k|,|j|=1, \ldots, N
$$

and

$$
\begin{aligned}
\int_{-T / 2}^{T / 2}\left|\sum_{|k|=1}^{N} a_{k} \mathrm{e}^{i \lambda_{k} t}+b_{k} \mathrm{e}^{i \mu_{k} t}\right|^{2} \mathrm{~d} & \geq \frac{2 T}{\pi}\left[\sum_{|k|=1}^{N} K_{T}(0)\left|a_{k}\right|^{2}\right. \\
& \left.-\left(\frac{2 \pi}{T \gamma}\right)^{2}\left(\sum_{|k|=1}^{N} \sum_{j \neq k}\left|a_{k}\right|\left|a_{j}\right| \frac{1}{4(k-j)^{2}-1}-2 \sum_{|k|,|j|=1}^{N}\left|a_{k}\right|\left|b_{j}\right| \frac{1}{4 d_{k, j}^{2}-1}\right)\right] .
\end{aligned}
$$

For the mixed terms, we compute

$$
\begin{gathered}
\sum_{|k|,|j|=1}^{N}\left|a_{k}\right|\left|b_{j}\right| \frac{1}{4 d_{k, j}^{2}-1} \leq \sum_{|k|,|j|=1}^{N}\left(\frac{M}{2}\left|a_{k}\right|^{2}+\frac{1}{2 M}\left|b_{j}\right|^{2}\right) \frac{1}{4 d_{k, j}^{2}-1}=\sum_{|k|=1}^{N}\left(\frac{M}{2}\left|a_{k}\right|^{2}+\frac{1}{2 M}\left|b_{k}\right|^{2}\right) \sum_{|j|=1}^{N} \frac{1}{4 d_{k, j}^{2}-1} \\
=\sum_{k=1}^{N}\left(\frac{M}{2}\left(\left|a_{k}\right|^{2}+\left|a_{-k}\right|^{2}\right)+\frac{1}{2 M}\left(\left|b_{k}\right|^{2}+\left|b_{-k}\right|^{2}\right)\right) \sum_{|j|=1}^{N} \frac{1}{4 d_{k, j}^{2}-1} \leq \sum_{k=1}^{N} M\left|a_{k}\right|^{2} \sum_{|j|=1}^{N} \frac{1}{4 d_{k, j}^{2}-1}
\end{gathered}
$$

We also have classically

$$
\sum_{|k|=1}^{N} \sum_{j \neq k}\left|a_{k}\right|\left|a_{j}\right| \frac{1}{4(k-j)^{2}-1} \leq \sum_{k=1}^{N}\left|a_{k}\right|^{2} \sum_{j \neq k}^{N} \frac{1}{4(k-j)^{2}-1}
$$

We then get

with

$$
\int_{-T / 2}^{T / 2}\left|\sum_{|k|=1}^{N} a_{k} \mathrm{e}^{i \lambda_{k} t}+b_{k} \mathrm{e}^{i \mu_{k} t}\right|^{2} \mathrm{~d} t \geq \frac{2 T}{\pi} \sum_{|k|=1}^{N}\left(\frac{2 \pi}{T \gamma}\right)^{2} c_{k}\left|a_{k}\right|^{2}
$$

$$
c_{k}:=\left(\frac{T \gamma}{2 \pi}\right)^{2} K_{T}(0)-\sum_{|j|=1, j \neq k}^{N} \frac{1}{4(k-j)^{2}-1}-2 M \sum_{j=1}^{N} \frac{1}{4 d_{k, j}^{2}-1} .
$$


We compute, for $k \geq N-N^{\alpha}$

$$
\begin{aligned}
c_{k}=\left(\frac{T \gamma}{2 \pi}\right)^{2}- & \left.\left(\sum_{j=-N}^{k-1}+\sum_{j=k+1}^{N}\right) \frac{1}{4(k-j)^{2}-1}-2 M \sum_{j=N-N^{\alpha}}^{N} \frac{1}{4(2 N+1-k-j)^{2}-1}\right)+C N^{1-2 \alpha} \\
= & \left(\frac{T \gamma}{2 \pi}\right)^{2}-o(1)-\left(\sum_{\ell=1}^{N+k}+\sum_{\ell=1}^{N-k}+2 M \sum_{\ell=N+1-k}^{N+1-k+N^{\alpha}}\right) \frac{1}{2}\left(\frac{-1}{2 \ell+1}+\frac{1}{2 \ell-1}\right) \\
& \left(\frac{T \gamma}{2 \pi}\right)^{2}-o(1)-\left(1+\left(M-\frac{1}{2}\right) \frac{1}{2(N-k)+1}\right)\left(\frac{T \gamma}{2 \pi}\right)^{2}-o(1)-\left(1, M+\frac{1}{2}\right),
\end{aligned}
$$

and we obtain similarly the same result for $k \leq-N+N^{\alpha}$, so that the proof is done, by taking $N$ large enough.

In order to prove Theorem 1.4, we give at first technical conditions that are satisfied, from the hypotheses.

Proposition 5.1. Suppose that the hypotheses of Theorem 1.4 are satisfied. Let $0<\varepsilon^{\prime}<\varepsilon, N \in \mathbb{N}^{*}$. The sequences $\left(\lambda_{k}\right)_{|k|=1}^{N},\left(\mu_{k}\right)_{|k|=1}^{N},\left(\gamma_{k}\right)_{|k|=1}^{N},\left(\gamma_{k}^{\prime}\right)_{|k|=1}^{N}$ are such that

(o) the hypotheses of Theorem 1.3 are satisfied;

(i) $\frac{\left|\lambda_{k}-\lambda_{j}\right|}{|k-j|} \geq \sqrt{\gamma_{k} \gamma_{j}}-c_{1} N^{-1 / 2-\varepsilon}$, for $0<|k-j| \leq N^{1 / 2+\varepsilon^{\prime}}$;

(ii) $\frac{\left|\mu_{k}-\mu_{j}\right|}{|k-j|} \geq \sqrt{\gamma_{k}^{\prime} \gamma_{j}^{\prime}}-c_{2} N^{-1 / 4-\varepsilon}$, for $0<|k-j| \leq N^{1 / 4+\varepsilon^{\prime}}$;

(iii) $\left|\mu_{k}-\mu_{j}\right| \geq c_{3}|k-j| \sqrt{\gamma_{k}^{\prime} \gamma_{j}^{\prime}}$, for $|k-j| \geq N^{1 / 4+\varepsilon^{\prime}}$;

(iv) $\gamma_{k}=\gamma_{-k}$ and $\gamma_{k}^{\prime}=\gamma_{-k}^{\prime}$ for $|k|=1, \ldots, N$;

(v) $\gamma_{k} \geq c_{4}>0$, for $|k|=1, \ldots, N$;

(vi) $\left|\gamma_{k}-\gamma_{j}\right| \leq c_{5}|k-j| h,\left|\gamma_{k}^{\prime}-\gamma_{j}^{\prime}\right| \leq c_{6}|k-j| h$, for $|k|,|j|=1, \ldots, N$;

where the constants $c_{1}, \ldots, c_{6}$ are independent of $N$, and $\gamma$ satisfies

$$
0<\gamma^{2} \leq \frac{\gamma_{k}^{2}+\gamma_{k}^{\prime 2}}{2}, \quad \gamma \leq \gamma_{k}
$$

Proof. We extend $f$ into an odd function, such that $f \in C^{3}[-1,1]$.

(o): it follows from the Taylor-Lagrange formula $\frac{f((k+1) h)-f(k h)}{h}=f^{\prime}(c), c \in(k h, k h+h)$;

(i): by using a Taylor development around the point $\frac{k+j}{2} h$, we get

$$
|| \frac{\lambda_{k}-\lambda_{j}}{k-j}\left|-f^{\prime}\left(\frac{k+j}{2} h\right)\right| \preceq|k-j|^{2} h^{2}, \quad\left|\sqrt{\gamma_{k} \gamma_{j}}-f^{\prime}\left(\frac{k+j}{2} h\right)\right| \preceq|k-j|^{2} h^{2} ;
$$

(ii): idem;

(iii): for $1 \geq x \geq 0$, we define $g(x)=f(1-\sqrt{1-x})$. Since $f^{\prime}(1)=0$, and $f^{\prime \prime}(1) \neq 0, g$ is differentiable at 1 and we have $g^{\prime}(1) \neq 0$. We define $g$ similarly on $[-1,0]$. We get

$$
\frac{\left|\mu_{k}-\mu_{j}\right|}{|k-j| \sqrt{\gamma_{k}^{\prime} \gamma_{j}^{\prime}}} \succeq \frac{|f(|N+1-k| h)-f(|N+1-j| h)|}{h^{2}|k-j||(|k|+|j|) / 2|},
$$

and by writing $\alpha=(N+1-k / 2) h, \beta=(N+1-j / 2) h$, we thus obtain

$$
\frac{\left|\mu_{k}-\mu_{j}\right|}{|k-j| \sqrt{\gamma_{k}^{\prime} \gamma_{j}^{\prime}}} \succeq \frac{\left|g\left(1-\left(1-\alpha^{2}\right)\right)-g\left(1-(1-\beta)^{2}\right)\right|}{|(\alpha-\beta)(2-\alpha-\beta)|} \succeq 1 ;
$$


(iv): by definition;

$(v)$ : because $\left|f^{\prime}(x)\right| \succeq 1$ for $x \in[0,1 / 2]$;

(vi): because $f \in C^{2}([-1,1])$.

In order to prove the main theorem, we will use again Ingham's first method, and we take the definition (33) of $K_{T}$.

Beginning of the proof of Theorem 1.4. We will prove that

$$
\int_{-T / 2}^{T / 2}\left|\sum_{|k|=1}^{N} \gamma_{k}^{2} a_{k} \mathrm{e}^{i \lambda_{k} t}+b_{k} \gamma_{k}^{\prime 2} \mathrm{e}^{i \mu_{k} t}\right|^{2} \mathrm{~d} t \succeq \sum_{|k|=1}^{N} \gamma_{k}^{4}\left|a_{k}\right|^{2}+\gamma_{k}^{\prime 4}\left|b_{k}\right|^{2}
$$

with sequences $\left(a_{k}\right),\left(b_{k}\right)$ satisfying $\left|b_{k}\right|^{2}+\left|b_{-k}\right|^{2} \leq\left|a_{k}\right|^{2}+\left|a_{-k}\right|^{2}$, and this will yield the result, by changing $a_{k}$ to $\gamma_{k}^{2} a_{k}$ and $b_{k}$ to $\gamma_{k}^{\prime 2} b_{k}$.

We take Ingham's first function used in Theorem 1.3, and we proceed at the beginning like in Theorem 1.3, but this time we do not get rid of the high frequency terms (34). We obtain:

$$
\begin{array}{r}
\int_{-T / 2}^{T / 2}\left|\sum_{|k|=1}^{N} \gamma_{k}^{2} a_{k} \mathrm{e}^{i \lambda_{k} t}+b_{k} \gamma_{k}^{\prime 2} \mathrm{e}^{i \mu_{k} t}\right|^{2} \mathrm{~d} t \succeq \sum_{|k|=1}^{N}\left(\left|a_{k}\right|^{2} \gamma_{k}^{4}+\left|b_{k}\right|^{2} \gamma_{k}^{\prime 4}\right)-\sum_{|k|,|j|=1, j \neq k}^{N}\left(\left|a_{k}\right|\left|a_{j}\right| \gamma_{k}^{2} \gamma_{j}^{2}\left|K_{T}\left(\lambda_{k}-\lambda_{j}\right)\right|\right. \\
\left.+\left|b_{k}\right|\left|b_{j}\right| \gamma_{k}^{\prime 2} \gamma_{j}^{\prime 2}\left|K_{T}\left(\mu_{k}-\mu_{j}\right)\right|\right)-B:=A-B, \quad B:=2 \sum_{|k|,|j|=1}^{N} \gamma_{k}^{2} \gamma_{j}^{\prime 2}\left|a_{k}\right|\left|b_{j}\right|\left|K_{T}\left(\lambda_{k}-\mu_{j}\right)\right| .
\end{array}
$$

By using the Young inequality

$$
\left|a_{k}\right|\left|a_{j}\right| \leq\left(\left|a_{k}\right|^{2}+\left|a_{j}\right|^{2}\right) / 2:=\tilde{a}_{k, j},\left|b_{k}\right|\left|b_{j}\right| \leq\left(\left|b_{k}\right|^{2}+\left|b_{j}\right|^{2}\right) / 2:=\tilde{b}_{k, j}
$$

we obtain for the first term

$$
\begin{aligned}
A \geq \sum_{|k|=1}^{N}\left(\left|a_{k}\right|^{2} \gamma_{k}^{4}+\left|b_{k}\right|^{2} \gamma_{k}^{\prime 4}\right) & -\sum_{k=1}^{N} \sum_{|j|=1, j \neq k}^{N}\left(\tilde{a}_{k, j} \gamma_{k}^{2} \gamma_{j}^{2}\left|K_{T}\left(\lambda_{k}-\lambda_{j}\right)\right|+\tilde{b}_{k, j} \gamma_{k}^{\prime 2} \gamma_{j}^{\prime 2}\left|K_{T}\left(\mu_{k}-\mu_{j}\right)\right|\right) \\
& -\sum_{k=1}^{N}\left(\sum_{|j|=1, j \neq k}^{N} \tilde{a}_{-k,-j} \gamma_{k}^{2} \gamma_{j}^{2}\left|K_{T}\left(\lambda_{-k}-\lambda_{-j}\right)\right|+\tilde{b}_{-k,-j} \gamma_{k}^{\prime 2} \gamma_{j}^{\prime 2}\left|K_{T}\left(\mu_{-k}-\mu_{-j}\right)\right|\right)
\end{aligned}
$$

because of $(i v)$.

From the notations $a_{k, j}:=\tilde{a}_{k, j}+\tilde{a}_{-k,-j}, K_{T}^{\lambda}(k, j):=\max \left(\left|K_{T}\left(\lambda_{k}-\lambda_{j}\right)\right|,\left|K_{T}\left(\lambda_{-k}-\lambda_{j}\right)\right|\right)$ and $K_{T}^{\mu}(k, j):=$ $\max \left(\left|K_{T}\left(\mu_{k}-\mu_{j}\right)\right|,\left|K_{T}\left(\mu_{-k}-\mu_{-j}\right)\right|\right)$, we get

$$
\begin{aligned}
& A \geq \sum_{k=1}^{N}\left(a_{k, k} \gamma_{k}^{4}+b_{k, k} \gamma_{k}^{\prime 4}\right)-\sum_{k=1}^{N} \sum_{|j|=1, j \neq k}^{N}\left(a_{k, j} \gamma_{k}^{2} \gamma_{j}^{2} K_{T}^{\lambda}(k, j)+b_{k, j} \gamma_{k}^{\prime 2} \gamma_{j}^{\prime 2} K_{T}^{\mu}(k, j)\right) \geq \sum_{k=1}^{N}\left(a_{k, k} \gamma_{k}^{4}+b_{k, k} \gamma_{k}^{\prime 4}\right) \\
& -\sum_{k=1}^{N} \sum_{|j|=1, j \neq k}^{N} c_{k, j}\left(a_{k, j} \gamma_{k}^{2} \gamma_{j}^{2}+b_{k, j} \gamma_{k}^{\prime 2} \gamma_{j}^{\prime 2}\right), \text { with } c_{k, j}:=\frac{a_{k, j} \gamma_{k}^{2} \gamma_{j}^{2} K_{T}^{\lambda}(k, j)+b_{k, j} \gamma_{k}^{\prime 2} \gamma_{j}^{\prime 2} K_{T}^{\mu}(k, j)}{a_{k, j} \gamma_{k}^{2} \gamma_{j}^{2}+b_{k, j} \gamma_{k}^{\prime 2} \gamma_{j}^{\prime 2}}, \text { for } j \neq k .
\end{aligned}
$$


Since we have $c_{k, j}=c_{j, k}=c_{-k,-j}$, we get

$$
\begin{gathered}
A-\sum_{k=1}^{N}\left(a_{k, k} \gamma_{k}^{4}+b_{k, k} \gamma_{k}^{\prime 4}\right) \geq-\sum_{k=1}^{N} \sum_{|j|=1, j \neq k}^{N} c_{k, j}\left(a_{k, k} \gamma_{k}^{2} \gamma_{j}^{2}+b_{k, k} \gamma_{k}^{\prime 2} \gamma_{j}^{\prime 2}\right)=-\sum_{k=1}^{N} \sum_{|j|=1, j \neq k}^{N} c_{k, j}\left(\frac{a_{k, k}+a_{j, j}}{2} \gamma_{k}^{2} \gamma_{j}^{2}\right. \\
\left.+\frac{b_{k, k}+b_{j, j}}{2} \gamma_{k}^{\prime 2} \gamma_{j}^{\prime 2}\right) \geq-\sum_{k=1}^{N} \sum_{j=1, j \neq k}^{N} c_{k, j}\left(a_{k, k} \gamma_{k}^{2} \gamma_{j}^{2}+b_{k, k} \gamma_{k}^{\prime 2} \gamma_{j}^{\prime 2}\right)-\sum_{k=1}^{N} \sum_{j=-N, j \neq k}^{-1} c_{k, j}\left(\frac{a_{k, k}+a_{j, j}}{2} \gamma_{k}^{2} \gamma_{j}^{2}\right. \\
\left.+\frac{b_{k, k}+b_{j, j}}{2} \gamma_{k}^{\prime 2} \gamma_{j}^{\prime 2}\right) \geq-\sum_{k=1}^{N} \sum_{j=1, j \neq k}^{N} c_{k, j}\left(a_{k, k} \gamma_{k}^{2} \gamma_{j}^{2}+b_{k, k} \gamma_{k}^{\prime 2} \gamma_{j}^{\prime 2}\right)-\sum_{k=1}^{N} \sum_{j=-N, j \neq k}^{-1} c_{k, j}\left(\frac{a_{k, k}}{2} \gamma_{k}^{2} \gamma_{j}^{2}+\frac{b_{k, k}}{2} \gamma_{k}^{\prime 2} \gamma_{j}^{\prime 2}\right) \\
-\sum_{j=-N}^{-1} \sum_{k=1, j \neq k}^{N} c_{k, j}\left(\frac{a_{k, k}}{2} \gamma_{k}^{2} \gamma_{j}^{2}+\frac{b_{k, k}}{2} \gamma_{k}^{\prime 2} \gamma_{j}^{\prime 2}\right) . A \geq \sum_{k=1}^{N}\left(a_{k, k} \gamma_{k}^{4}+b_{k, k} \gamma_{k}^{\prime 4}\right)\left(1-\sum_{|j|=1, j \neq k}^{N} c_{k, j} \frac{a_{k, k} \gamma_{k}^{2} \gamma_{j}^{2}+b_{k, k} \gamma_{k}^{\prime 2} \gamma_{j}^{\prime 2}}{a_{k, k} \gamma_{k}^{4}+b_{k, k} \gamma_{k}^{\prime 4}}\right) .
\end{gathered}
$$

We now consider

$$
\Phi(x)=\frac{\gamma_{k}^{2} \gamma_{j}^{2}+x \gamma_{k}^{\prime 2} \gamma_{j}^{\prime 2}}{\gamma_{k}^{4}+x \gamma_{k}^{\prime 4}}
$$

Since $b_{k, k} \leq a_{k, k}$, we have $\Phi\left(\frac{b_{k, k}}{a_{k, k}}\right) \leq \max (\Phi(0), \Phi(1))$, and thus

$$
A \geq \sum_{k=1}^{N}\left(a_{k, k} \gamma_{k}^{4}+b_{k, k} \gamma_{k}^{4}\right)\left(1-\sum_{|j|=1, j \neq k}^{N} c_{k, j} \gamma_{k, j}\right), \text { with } \gamma_{k, j}:=\max \left(\frac{\gamma_{k}^{2} \gamma_{j}^{2}+\gamma_{k}^{\prime 2} \gamma_{j}^{\prime 2}}{\gamma_{k}^{4}+\gamma_{k}^{\prime 4}}, \frac{\gamma_{j}^{2}}{\gamma_{k}^{2}}\right)
$$

In order to estimate the term $c_{k, j}$ which mixes the high and the low frequencies, we define the function

$$
g(x)=\min \left(1, \frac{1}{|x-1|}\right)
$$

and we can check the following property:

Lemma 5.2. The function $g: \mathbb{R}^{+} \rightarrow \mathbb{R}^{+}$satisfies

- $\left|K_{T}(x)\right| \leq g_{T}(x):=g\left(\frac{T^{2} x^{2}}{\pi^{2}}\right), x \in \mathbb{R}^{+}$;

- $g$ is decreasing;

- $g$ satisfies the following estimate:

$$
x^{2} g(x)+y^{2} g(y) \leq\left(x^{2}+y^{2}\right) g\left(\frac{x+y}{2}\right), \quad x, y \in \mathbb{R}^{+} .
$$

Proof. The second point is clear. For the first point it suffices to prove that

$$
\left|\cos \left(\frac{\pi}{2} x\right)\right| \leq\left|1-x^{2}\right|, \quad x \in \mathbb{R}^{+},
$$

and this can be done by computing the variations of $\psi: x \rightarrow 1-x^{2}-\cos \left(\frac{\pi}{2} x\right)$, for $0 \leq x \leq 1$ (we have $\psi^{(3)} \leq 0$, $\psi^{(2)}(0)=-2+\left(\frac{\pi}{2}\right)^{2}>0, \psi^{(2)}(1)=-2<0 \psi^{\prime}(0)=0, \psi^{\prime}(1)=-2+\frac{\pi}{2}<0, \psi(0)=0$ and $\left.\psi(1)=0\right)$, the variations of $\chi=-\psi$, for $1 \leq x \leq \sqrt{2}$ (we have $\chi^{(3)} \geq 0, \chi^{(2)}(1)>0, \chi^{\prime}(1)>0$ and $\chi(1)=0$ ), and by remarking that $\left|\cos \left(\frac{\pi}{2} x\right)\right| \leq 1 \leq\left|1-x^{2}\right|$, for $x \geq \sqrt{2}$. 
For the last point, by symmetry, it suffices to consider the following three cases.

(i) If $x \leq 2, y \leq 2$, we have $g(x)=g(y)=g\left(\frac{x+y}{2}\right)$, and we get the result.

(ii) If $x \geq 2, y \geq 2$, the function $\psi: t \rightarrow \frac{t^{2}}{t-1}$ is increasing for $t \geq 2\left(\psi^{\prime}(t)=\frac{t(t-2)}{(t-1)^{2}} \geq 0\right)$, and thus we get

$$
2\left(x^{2}+y^{2}\right)-(x+y-2)\left(\frac{x^{2}}{x-1}+\frac{y^{2}}{y-1}\right)=x^{2}+y^{2}-(y-1) \frac{x^{2}}{x-1}-(x-1) \frac{y^{2}}{y-1}=(x-y)\left(\frac{x^{2}}{x-1}-\frac{y^{2}}{y-1}\right) \geq 0 .
$$

This yields the result.

(iii) If $x \leq 2, y \geq 2$ (the case $x \geq 2, y \leq 2$ can be treated by symmetry), we have

$$
\begin{aligned}
& 2 x^{2}+2 y^{2}-(x+y-2)\left(x^{2}+\frac{y^{2}}{y-1}\right)=x^{2}(2+2-x-y)+\frac{y^{2}}{y-1}(2 y-2+2-x-y) \\
= & \left(x^{2}+\frac{y^{2}}{y-1}\right)(2-x)+\left(x^{2}-4\right)(2-y)+4(2-y)+\frac{y^{2}}{y-1}(y-2) \geq(y-2)\left(-4+\frac{y^{2}}{y-1}\right)=\frac{(y-2)^{3}}{y-1} \geq 0
\end{aligned}
$$

and the proof is completed.

The following lemma gives a sharper estimate, and allows us to improve the observability time in the applications.

Lemma 5.3. If $x, y \in \mathbb{R}^{+}$satisfy $x+y-\sqrt{x y} \geq 4$, then we have

$$
x^{2} g(x)+y^{2} g(y) \leq\left(x^{2}+y^{2}\right) g(x+y-\sqrt{x y}) \leq\left(x^{2}+y^{2}\right) g\left(\frac{x+y}{2}\right) .
$$

Proof. The right hand side comes from the decreasingness of $g$ and from the Young inequality $x y \leq \frac{x^{2}+y^{2}}{2}$.

We distinguish three cases:

(i) If $x \geq 3, y \geq 3$, then the function $\psi: t \rightarrow \frac{t^{3 / 2}}{t-1}$ is increasing $\left(\psi^{\prime}(t)=\frac{\sqrt{t}(t-3)}{2(t-1)^{2}} \geq 0\right)$, and thus, using (38), we get

$$
\begin{gathered}
2\left(x^{2}+y^{2}\right)-2(x+y-\sqrt{x y}-1)\left(\frac{x^{2}}{x-1}+\frac{y^{2}}{y-1}\right)=2\left(x^{2}+y^{2}\right)-\left(x+y-2+(\sqrt{x}-\sqrt{y})^{2}\right)\left(\frac{x^{2}}{x-1}+\frac{y^{2}}{y-1}\right) \\
=(x-y)\left(\frac{x^{2}}{x-1}-\frac{y^{2}}{y-1}\right)-(\sqrt{x}-\sqrt{y})^{2}\left(\frac{x^{2}}{x-1}+\frac{y^{2}}{y-1}\right) \\
=(\sqrt{x}-\sqrt{y})\left((\sqrt{x}+\sqrt{y})\left(\frac{x^{2}}{x-1}-\frac{y^{2}}{y-1}\right)-(\sqrt{x}-\sqrt{y})\left(\frac{x^{2}}{x-1}+\frac{y^{2}}{y-1}\right)\right)=2(\sqrt{x}-\sqrt{y}) \sqrt{x y}\left(\frac{x^{3 / 2}}{x-1}-\frac{y^{3 / 2}}{y-1}\right) \geq 0 .
\end{gathered}
$$

(ii) If $2 \leq x \leq 3$ (the case $2 \leq y \leq 3$ can be treated by symmetry), since $y-\sqrt{x} \sqrt{y}+x-4 \geq 0$ and $0 \leq x \leq 4$, we have

$$
\sqrt{y} \geq \frac{\sqrt{x}+\sqrt{16-3 x}}{2}=: \sqrt{z_{0}}(x) .
$$

We can notice that the function $z_{0}$ is decreasing for $x \in(2,3)$, and thus $y \geq z_{0}(x) \geq z_{0}(3)=\left(\frac{\sqrt{3}+\sqrt{7}}{2}\right)^{2} \geq 4$ and $x \leq y$. Therefore, we infer from (i) that $\psi(y) \geq \psi\left(z_{0}(x)\right)$ and it remains to prove that

$$
\psi\left(z_{0}(x)\right)-\psi(x) \geq 0 .
$$

Setting $z:=z_{0}(x)$, for brevity, we have

$$
z^{3 / 2}(x-1)-x^{3 / 2}(z-1)=(\sqrt{z}-\sqrt{x}) x z+x^{3 / 2}-z^{3 / 2}=(\sqrt{z}-\sqrt{x})(x z-x-z-\sqrt{x z})=(\sqrt{z}-\sqrt{x})(x z-2 \sqrt{x z}-4),
$$


because $x+z-\sqrt{x z}=4$. We notice that $x \rightarrow \sqrt{x y}$ is increasing, so that $\sqrt{x y} \geq \sqrt{2 z_{0}(2)}=1+\sqrt{5}$, and thus $x z-2 \sqrt{x z}-4$ is positive. From the previous computation, we finally obtain (39).

(iii) If $x \leq 2$ (the case $y \leq 2$ can be treated similarly by symmetry), we notice that $z_{0}$ is increasing for $0 \leq x \leq 4 / 3$, and decreasing for $4 / 3 \leq x \leq 2$, and we have $y \geq 4$, so that $\psi\left(z_{0}\right)$ varies like $y$, for $0 \leq x \leq 2$. Now, if $0 \leq x \leq 4 / 3$, we have

$$
\frac{y^{3 / 2}}{y-1}=\psi(y) \geq \psi\left(z_{0}(x)\right) \geq \psi\left(z_{0}(0)\right)=\frac{8}{3} \geq\left(\frac{4}{3}\right)^{3 / 2} \geq x^{3 / 2} .
$$

On the other hand, if $4 / 3 \leq x \leq 2$, we obtain also $\frac{y^{3 / 2}}{y-1} \geq \psi\left(z_{0}(2)\right)=2^{3 / 2} \geq x^{3 / 2}$. We thus get

$$
\begin{aligned}
& 2\left(x^{2}+y^{2}\right)-2(x+y-\sqrt{x y}-1)\left(x^{2}+\frac{y^{2}}{y-1}\right)=2\left(x^{2}+y^{2}\right)-\left(x+y-2+(\sqrt{x}-\sqrt{y})^{2}\right)\left(x^{2}+\frac{y^{2}}{y-1}\right)=x^{2}(4-x-y) \\
+ & \frac{y^{2}}{y-1}(y-x)-(\sqrt{x}-\sqrt{y})^{2}\left(x^{2}+\frac{y^{2}}{y-1}\right)=x^{2}(4-2 x)+(x-y)\left(x^{2}-\frac{y^{2}}{y-1}\right)-(\sqrt{x}-\sqrt{y})^{2}\left(x^{2}+\frac{y^{2}}{y-1}\right)=x^{2}(4-2 x) \\
+ & (\sqrt{x}-\sqrt{y})\left((\sqrt{x}+\sqrt{y})\left(x^{2}-\frac{y^{2}}{y-1}\right)-(\sqrt{x}-\sqrt{y})\right)\left(x^{2}+\frac{y^{2}}{y-1}\right)=x^{2}(4-2 x)+2(\sqrt{y}-\sqrt{x}) \sqrt{x y}\left(\frac{y^{3 / 2}}{y-1}-x^{3 / 2}\right) \geq 0,
\end{aligned}
$$

so that we conclude the proof.

The next lemma gives the useful estimates for the errors with the approximative case where we have $\left|\lambda_{k}-\lambda_{j}\right| \geq$ $\left|(k-j) \sqrt{\gamma_{k} \gamma_{j}}\right|$ and $\left|\mu_{k}-\mu_{j}\right| \geq\left|(k-j) \sqrt{\gamma_{k}^{\prime} \gamma_{j}^{\prime}}\right|$, to which we will apply the preceding lemma. For convenience, we will write $e_{s}$ for a sequence depending on $j, k, N$ and satisfying

$$
\sum_{|j|=1}^{N} e_{s} \preceq N^{-\varepsilon_{s}}, \quad \varepsilon_{s}>0 .
$$

Lemma 5.4. With the hypotheses (o)-(iv) of Theorem 1.4, and for $N$ large enough, we have

$$
\left|K_{T}\left(\lambda_{k}-\lambda_{j}\right)\right| \leq g_{T}\left((k-j) \sqrt{\gamma_{k} \gamma_{j}}\right)+e_{s},
$$

and

$$
\left(\gamma_{k}^{\prime} \gamma_{j}^{\prime}\right)^{2}\left|K_{T}\left(\mu_{k}-\mu_{j}\right)\right| \leq\left(\gamma_{k}^{\prime} \gamma_{j}^{\prime}\right)^{2} g_{T}\left((k-j) \sqrt{\gamma_{k}^{\prime} \gamma_{j}^{\prime}}\right)+\max \left(1,\left(\gamma_{k}^{\prime} \gamma_{j}^{\prime}\right)^{2}\right) e_{s}
$$

Proof. We first consider (40). Suppose at first that $|k-j|>N^{1 / 2+\varepsilon}$, then we have from assumption (o) that

$$
\left|\lambda_{k}-\lambda_{j}\right| \succeq|k-j| \succeq N^{1 / 2+\varepsilon}
$$

and thus

Now, if $0<|k-j| \leq N^{1 / 2+\varepsilon}$, we define

$$
\left|K_{T}\left(\lambda_{k}-\lambda_{j}\right)\right| \preceq N^{-1-\varepsilon}=: e_{1} .
$$

$$
e_{s}:=\left|g_{T}\left(|k-j| \sqrt{\gamma_{k} \gamma_{j}}\right)-g_{T}\left(|k-j|\left(\sqrt{\gamma_{k} \gamma_{j}}-c_{1} N^{-1 / 2-\varepsilon}\right)\right)\right| .
$$

Since $\gamma \leq \sqrt{\gamma_{k} \gamma_{j}}$ and $T>2 \pi / \gamma$, we obtain

$$
e_{s} \preceq \frac{|k-j|}{N^{1 / 2+\varepsilon}} \cdot \frac{1}{|k-j|^{3}} \preceq \frac{1}{N^{1 / 2+\varepsilon}|k-j|^{2}},
$$


using that $x^{3} g_{T}^{\prime}(x)$ is bounded for $|x-| k-j\left|\sqrt{\gamma_{k} \gamma_{j}}\right| \preceq N^{-1 / 2-\varepsilon}$, and $N$ large enough. By taking $e_{s}=0$, for $k=j$, we get (40).

We now consider (41). We define

$$
e_{s}:=\left(\gamma_{k}^{\prime} \gamma_{j}^{\prime}\right)^{2}\left|g_{T}\left(|k-j| \sqrt{\gamma_{k}^{\prime} \gamma_{j}^{\prime}}\right)-g_{T}\left(|k-j|\left(\sqrt{\gamma_{k}^{\prime} \gamma_{j}^{\prime}}-c_{2} N^{-1 / 4-\varepsilon}\right)\right)\right|,
$$

for $0<|k-j| \leq N^{1 / 4+\varepsilon^{\prime}}$.

We define $\delta_{k, j}:=|k-j|\left(\sqrt{\gamma_{k}^{\prime} \gamma_{j}^{\prime}}-c_{2} N^{-1 / 4-\varepsilon}\right)$. Since $c_{2} \geq 0$, we always have $\delta_{k, j} \leq|k-j| \sqrt{\gamma_{k}^{\prime} \gamma_{j}^{\prime}}$.

Now, if $\delta_{k, j} \leq 0$, we obtain $e_{s} \preceq\left(\gamma_{k}^{\prime} \gamma_{j}^{\prime}\right)^{2} \preceq N^{-1-4 \varepsilon}$.

If $0 \leq \delta_{k, j} \leq \sqrt{\gamma_{k}^{\prime} \gamma_{j}^{\prime}}|k-j| \leq \sqrt{2} \frac{\pi}{T}$, we have

$$
1=g_{T}(0) \geq g_{T}\left(\delta_{k, j}\right) \geq g_{T}\left(\sqrt{\gamma_{k}^{\prime} \gamma_{j}^{\prime}}|k-j|\right) \geq g_{T}\left(\sqrt{2} \frac{\pi}{T}\right)=1
$$

and thus $e_{s}=0$

If $0 \leq \delta_{k, j} \leq \sqrt{2} \frac{\pi}{T} \leq \sqrt{\gamma_{k}^{\prime} \gamma_{j}^{\prime}}|k-j|$, we have

$$
\left|g_{T}\left(|k-j| \sqrt{\gamma_{k}^{\prime} \gamma_{j}^{\prime}}\right)-1\right| \preceq\left|\sqrt{2} \frac{\pi}{T}-\right| k-j\left|\sqrt{\gamma_{k}^{\prime} \gamma_{j}^{\prime}}\right| \preceq|k-j| N^{-1 / 4-\varepsilon},
$$

and thus,

Since $0 \leq \delta_{k, j} \leq \sqrt{2} \frac{\pi}{T}$, we obtain

$$
e_{s} \preceq\left(\gamma_{k}^{\prime} \gamma_{j}^{\prime}\right)^{2}|k-j| N^{-1 / 4-\varepsilon} \preceq\left(\gamma_{k}^{\prime} \gamma_{j}^{\prime}\right)^{2} N^{\varepsilon^{\prime}-\varepsilon} .
$$

$$
\left(\gamma_{k}^{\prime} \gamma_{j}^{\prime}\right)^{2} \preceq\left(\frac{1}{|k-j|}+\frac{1}{N^{1 / 4+\varepsilon}}\right)^{4} \preceq \frac{1}{|k-j|^{4}}+\frac{1}{N}
$$

which yields

$$
e_{s} \preceq\left(\frac{1}{|k-j|^{4}}+\frac{1}{N}\right) N^{\varepsilon^{\prime}-\varepsilon} .
$$

It remains the case where $|k-j| \sqrt{\gamma_{k}^{\prime} \gamma_{j}^{\prime}} \geq \delta_{k, j} \geq \sqrt{2} \frac{\pi}{T}$. We subdivide it in two subcases. Suppose first that $\left|\gamma_{k}^{\prime} \gamma_{j}^{\prime}\right| \leq N^{-1 / 8-\varepsilon^{\prime} / 2}$. We first have

$$
\left|g_{T}\left(|k-j| \sqrt{\gamma_{k}^{\prime} \gamma_{j}^{\prime}}\right)-g_{T}\left(\delta_{k, j}\right)\right| \preceq N^{-1 / 4-\varepsilon}|k-j| \preceq N^{\varepsilon^{\prime}-\varepsilon},
$$

and thus

$$
N^{1 / 4+\varepsilon^{\prime}} e_{s} \preceq N^{1 / 4+\varepsilon^{\prime}}\left(\gamma_{k}^{\prime} \gamma_{j}^{\prime}\right)^{2} N^{\varepsilon^{\prime}-\varepsilon} \preceq N^{\varepsilon^{\prime}-\varepsilon} .
$$

Now, if we have instead $\left|\gamma_{k}^{\prime} \gamma_{j}^{\prime}\right| \geq N^{-1 / 8-\varepsilon^{\prime} / 2}$, we obtain $\sqrt{\gamma_{k}^{\prime} \gamma_{j}^{\prime}} \geq N^{-1 / 4-\varepsilon^{\prime} / 4}$, and then

$$
\delta_{k, j} / \sqrt{\gamma_{k}^{\prime} \gamma_{j}^{\prime}} \succeq 1-N^{-1 / 4-\varepsilon} / \sqrt{\gamma_{k}^{\prime} \gamma_{j}^{\prime}} \succeq 1-|k-j| N^{\varepsilon^{\prime} / 4-\varepsilon} \succeq 1,
$$

which yields $\delta_{k, j} \succeq \sqrt{\gamma_{k}^{\prime} \gamma_{j}^{\prime}}$, and thus

$$
e_{s} \preceq\left(\gamma_{k}^{\prime} \gamma_{j}^{\prime}\right)^{2}|k-j| N^{-1 / 4-\varepsilon} \frac{1}{|k-j|^{3}\left(\gamma_{k}^{\prime} \gamma_{j}^{\prime}\right)^{3 / 2}} \preceq \sqrt{\gamma_{k}^{\prime} \gamma_{j}^{\prime}} N^{-1 / 4-\varepsilon} \frac{1}{|k-j|^{2}},
$$

which gives the result, by changing $e_{s}$ into $e_{s} /\left(\max 1,\left(\gamma_{k}^{\prime} \gamma_{j}^{\prime}\right)^{2}\right)$. 
It remains now to consider the case where $|k-j| \geq N^{1 / 4+\varepsilon^{\prime}}$. We suppose now that $|k-j| \leq N^{\alpha}$; we will prove that we can find $e_{s}$, for $|k-j| \geq N^{3 / 4 \alpha+\tilde{\varepsilon}}$, with a number $\tilde{\varepsilon} \leq 1 / 16$. This will yield the result, since we can begin with $\alpha=1$ and obtain by recurrence $\alpha=\left(\frac{3}{4}\right)^{n}(1-4 \tilde{\varepsilon})+4 \tilde{\varepsilon}$, which will then be smaller $1 / 4+\varepsilon^{\prime}$, for $n$ large enough.

We suppose therefore that $N^{3 / 4 \alpha+\tilde{\varepsilon}} \leq|k-j| \leq N^{\alpha}$. Now, if $\left|\mu_{k}-\mu_{j}\right| \leq N^{\alpha / 2+\tilde{\varepsilon}}$, we have

$$
N^{\alpha}\left|K_{T}\left(\mu_{k}-\mu_{j}\right)\right| \preceq N^{\alpha} N^{-2(\alpha / 2+\tilde{\varepsilon})} \preceq N^{-2 \tilde{\varepsilon}},
$$

which ensures the existence of $e_{s}$. On the other hand, if $\left|\mu_{k}-\mu_{j}\right| \leq N^{\alpha / 2+\tilde{\varepsilon}}$, we then have

$$
N^{\alpha}\left(\gamma_{k}^{\prime} \gamma_{j}^{\prime}\right)^{2}\left|K_{T}\left(\mu_{k}-\mu_{j}\right)\right| \preceq N^{\alpha}\left(\sqrt{\gamma_{k}^{\prime} \gamma_{j}^{\prime}}\right)^{4} \preceq \frac{N^{3 \alpha+2 \tilde{\varepsilon}}}{|k-j|^{4}} \preceq N^{-2 \tilde{\varepsilon}},
$$

from assumption (iii), which ensures the existence of $e_{s}$ and this ends the proof.

Now, we can estimate the term $c_{k, j}$.

Lemma 5.5. We have for $j \neq k$

$$
\left|c_{k, j}\right| \leq e_{s}+\frac{1}{\left|\frac{T^{2}}{\pi^{2}}(k-j)^{2} \gamma^{2}-1\right|}
$$

Proof. We have at first

$$
\left|c_{k, j}\right| \leq \frac{a_{k, j} \gamma_{k}^{2} \gamma_{j}^{2} g_{T}\left((k-j) \sqrt{\gamma_{k} \gamma_{j}}\right)+b_{k, j} \gamma_{k}^{\prime 2} \gamma_{j}^{\prime 2} g_{T}\left((k-j) \sqrt{\gamma_{k}^{\prime} \gamma_{j}^{\prime}}\right)}{a_{k, j} \gamma_{k}^{2} \gamma_{j}^{2}+b_{k, j} \gamma_{k}^{\prime 2} \gamma_{j}^{\prime 2}}+B_{1}:=A_{1}+B_{1},
$$

with $B_{1}:=e_{s} \frac{a_{k, j} \gamma_{k}^{2} \gamma_{j}^{2}+b_{k, j} \max \left(1,\left(\gamma_{k}^{\prime} \gamma_{j}^{\prime}\right)^{2}\right)}{a_{k, j} \gamma_{k}^{2} \gamma_{j}^{2}+b_{k, j} \gamma_{k}^{\prime 2} \gamma_{j}^{\prime 2}}$. Now, for $B_{1}$, if $\gamma_{k}^{\prime} \gamma_{j}^{\prime} \leq 1$, we obtain

$$
B_{1} \preceq e_{s} \frac{a_{k, j}\left(\gamma_{k}^{2} \gamma_{j}^{2}+1\right)}{a_{k, j} \gamma_{k}^{2} \gamma_{j}^{2}} \preceq e_{s},
$$

since $b_{k, j} \leq a_{k, j}$, and $\gamma_{k} \succeq 1$, from assumption $(v)$. On the other hand, if $\gamma_{k}^{\prime} \gamma_{j}^{\prime} \geq 1$, we have

$$
B_{1} \preceq e_{s}\left(\frac{a_{k, j} \gamma_{k}^{2} \gamma_{j}^{2}}{a_{k, j} \gamma_{k}^{2} \gamma_{j}^{2}}+\frac{b_{k, j} \gamma_{k}^{\prime 2} \gamma_{j}^{\prime 2}}{b_{k, j} \gamma_{k}^{\prime 2} \gamma_{j}^{\prime 2}}\right) \preceq e_{s} .
$$

Concerning $A_{1}$, we have at first $A_{1}=\Phi\left(b_{k, j} / a_{k, j}\right)$, with

$$
\Phi(z)=\frac{\gamma_{k}^{2} \gamma_{j}^{2} g_{T}\left((k-j) \sqrt{\gamma_{k} \gamma_{j}}\right)+z \gamma_{k}^{\prime} \gamma_{j}^{\prime} g_{T}\left((k-j) \sqrt{\gamma_{k}^{\prime} \gamma_{j}^{\prime}}\right)}{\gamma_{k}^{2} \gamma_{j}^{2}+z \gamma_{k}^{\prime 2} \gamma_{j}^{\prime 2}} .
$$

Since $b_{k, j} \leq a_{k, j}$, we have $\Phi(z) \leq \max (\Phi(0), \Phi(1))$. As $\gamma \leq \gamma_{k}$, we already have

$$
\Phi(0) \leq \frac{1}{\left|\frac{T^{2}}{\pi^{2}}(k-j)^{2} \gamma^{2}-1\right|} .
$$

Concerning $\Phi(1)$, we use at first formula (37) with $x=\left(\frac{T}{\pi}\right)^{2}(k-j)^{2} \gamma_{k} \gamma_{j}$ and $y=\left(\frac{T}{\pi}\right)^{2}(k-j)^{2} \gamma_{k}^{\prime} \gamma_{j}^{\prime}$, so that we obtain

$$
\Phi(1) \leq \frac{1}{\left|\left(\frac{T}{2 \pi}\right)^{2}(k-j)^{2}\left(\gamma_{k} \gamma_{j}+\gamma_{k}^{\prime} \gamma_{j}^{\prime}\right)-1\right|} .
$$


Now, if $|k-j| \geq N^{1 / 2+\varepsilon_{s}}$, with a number $\varepsilon_{s}>0$, we get

$$
N \Phi(1) \preceq \frac{N}{|k-j|^{2}} \preceq N^{-\varepsilon_{s}} .
$$

On the other hand, if $|k-j| \leq N^{1 / 2+\varepsilon_{s}}$, we define

$$
\psi(x)=\frac{1}{\left|\left(\frac{T}{\pi}\right)^{2}(k-j)^{2}\left(\frac{\gamma_{k}^{2}+\gamma_{k}^{\prime 2}}{2}\right) x-1\right|} .
$$

We then have

$$
\Phi(1) \leq \frac{1}{\left|\frac{T^{2}}{\pi^{2}}(k-j)^{2} \gamma^{2}-1\right|}+\left|\psi(1)-\psi\left(x_{0}\right)\right|, \text { with } x_{0}:=\frac{\gamma_{k} \gamma_{j}+\gamma_{k}^{\prime} \gamma_{j}^{\prime}}{\gamma_{k}^{2}+\gamma_{k}^{\prime 2}}
$$

Now we have

$$
\left|x_{0}-1\right| \leq \frac{\gamma_{k}\left|\gamma_{j}-\gamma_{k}\right|}{\gamma_{k}^{2}+\gamma_{k}^{\prime 2}}+\frac{\gamma_{k}^{\prime}\left|\gamma_{k}^{\prime}-\gamma_{j}^{\prime}\right|}{\gamma_{k}^{2}+\gamma_{k}^{\prime 2}} \leq \frac{\gamma_{k}+\gamma_{k}^{\prime}}{\gamma_{k}^{2}+\gamma_{k}^{\prime 2}}|k-j| h \leq \frac{1}{\left|\gamma_{k}\right|} \frac{1+\frac{\gamma_{k}^{\prime}}{\gamma_{k}}}{1+\left(\frac{\gamma_{k}^{\prime}}{\gamma_{k}}\right)^{2}}|k-j| h \preceq|k-j| h,
$$

by using assumption $(v i)$. Computing the derivative, we get $\left|\psi^{\prime}(x)\right| \preceq \frac{1}{|k-j|^{2}}$, for $x \succeq 1$, so that

$$
\Phi(1) \leq \frac{1}{\left|\frac{T^{2}}{\pi^{2}}(k-j)^{2} \gamma^{2}-1\right|}+\frac{C}{|k-j|^{2}}|k-j| h \leq \frac{1}{\left|\frac{T^{2}}{\pi^{2}}(k-j)^{2} \gamma^{2}-1\right|}+C h
$$

by taking $\varepsilon_{s}$ small enough, so that $x_{0} \succeq 1$. We thus have

$$
\left|c_{k, j}\right| \leq \frac{1}{\left|\frac{T^{2}}{\pi^{2}}(k-j)^{2} \gamma^{2}-1\right|}+e_{s}, \quad k \neq j
$$

We also have an estimate for $\gamma_{k, j}$.

Lemma 5.6. We have $\gamma_{k, j} \leq 1+C|k-j| h \preceq 1$.

Proof. We compute

$$
\frac{\gamma_{j}^{2}}{\gamma_{k}^{2}} \leq 1+\frac{\left|\gamma_{j}-\gamma_{k}\right|}{\gamma_{k}} \frac{\left|\gamma_{k}+\gamma_{j}\right|}{\gamma_{k}} \leq 1+\frac{\left|\gamma_{j}-\gamma_{k}\right|}{\gamma_{k}}\left(2+\frac{\left|\gamma_{j}-\gamma_{k}\right|}{\gamma_{k}}\right)
$$

Now, since $\left|\gamma_{k}-\gamma_{j}\right| \preceq|k-j| h$, and since $\gamma_{k} \succeq 1$, we get $\frac{\gamma_{j}^{2}}{\gamma_{k}^{2}} \leq 1+C|k-j| h \preceq 1$. We compute again

$$
\begin{aligned}
& \frac{\gamma_{k}^{2} \gamma_{j}^{2}+\gamma_{k}^{\prime 2} \gamma_{j}^{\prime 2}}{\gamma_{k}^{4}+\gamma_{k}^{\prime 4}} \leq 1+\frac{\gamma_{k}^{2}\left|\gamma_{j}+\gamma_{k}\right|\left|\gamma_{j}-\gamma_{k}\right|}{\gamma_{k}^{4}+\gamma_{k}^{\prime 4}}+\frac{\gamma_{k}^{\prime 2}\left|\gamma_{j}^{\prime}+\gamma_{k}^{\prime}\right|\left|\gamma_{j}^{\prime}-\gamma_{k}^{\prime}\right|}{\gamma_{k}^{4}+\gamma_{k}^{\prime 4}} \leq \\
& 1+C \frac{\left(\gamma_{k}^{3}+\gamma_{k}^{\prime 3}\right)|k-j| h+\left(\gamma_{k}^{2}+\gamma_{k}^{\prime 2}\right)|k-j|^{2} h^{2}}{\gamma_{k}^{4}+\gamma_{k}^{\prime 4}},
\end{aligned}
$$

since $\left|\gamma_{k}-\gamma_{j}\right| \preceq|k-j| h$ and $\left|\gamma_{k}^{\prime}-\gamma_{j}^{\prime}\right| \preceq|k-j| h$. From $\gamma_{k} \succeq 1$, we obtain

$$
\frac{\gamma_{k}^{3}+\gamma_{k}^{\prime 3}}{\gamma_{k}^{4}+\gamma_{k}^{\prime 4}}=\frac{1}{\gamma_{k}} \frac{1+\left(\frac{\gamma_{k}^{\prime}}{\gamma_{k}}\right)^{3}}{+\left(\frac{\gamma_{k}^{\prime}}{\gamma_{k}}\right)^{4}} \preceq 1, \text { and similarly, } \frac{\gamma_{k}^{2}+\gamma_{k}^{\prime 2}}{\gamma_{k}^{4}+\gamma_{k}^{\prime 4}} \preceq 1
$$

which ends the proof. 
Proof of Theorem 1.4. We have $A \geq \sum_{k=1}^{N}\left(\gamma_{k}^{4} a_{k, k}+\gamma_{k}^{\prime 4} b_{k, k}\right)\left(1-d_{k}\right)$, with

$$
\begin{aligned}
d_{k}:=\sum_{|j|=1, j \neq k}^{N}\left(\frac{1}{\left|\frac{T^{2}}{\pi^{2}}(k-j)^{2} \gamma^{2}-1\right|}+e_{s}\right)(1+C|k-j| h) \leq \\
\qquad\left(\sum_{|j|=1, j \neq k}^{N} \frac{1}{\left|\frac{T^{2}}{\pi^{2}}(k-j)^{2} \gamma^{2}-1\right|}+C \sum_{|j|=1, j \neq k}^{N} \frac{|k-j| h}{(k-j)^{2}}\right)+o(1) .
\end{aligned}
$$

Since $\sum_{|j|=1, j \neq k}^{N} \frac{|k-j| h}{(k-j)^{2}} \leq \sum_{|j|=1}^{2 N} \frac{h}{j}=o(1)$, we have

$$
d_{k} \leq\left(\sum_{|j|=1, j \neq k}^{N} \frac{1}{\left|\frac{T^{2}}{\pi^{2}}(k-j)^{2} \gamma^{2}-1\right|}\right)+o(1) \leq\left(\frac{2 \pi}{T \gamma}\right)^{2}\left(\sum_{|j|=1, j \neq k}^{N} \frac{1}{\left|4(k-j)^{2}-1\right|}\right)+o(1) .
$$

Now, from the proof of Theorem 1.3, we have

$$
\begin{aligned}
& B \leq 2 \sum_{|k|,|j|=1}^{N} \gamma_{k}^{2} \gamma_{j}^{\prime 2}\left|a_{k}\right|\left|b_{j}\right|\left(\frac{2 \pi}{T \gamma}\right)^{2} \frac{1}{4 d_{k, j}^{2}-1} \leq \sum_{|k|,|j|=1}^{N}\left(\gamma_{k}^{4}\left|a_{k}\right|^{2}+\gamma_{j}^{\prime 4}\left|b_{j}\right|^{2}\right)\left(\frac{2 \pi}{T \gamma}\right)^{2} \frac{1}{4 d_{k, j}^{2}-1} \\
& =\sum_{|k|=1}^{N}\left(\gamma_{k}^{4}\left|a_{k}\right|^{2}+\gamma_{k}^{\prime 4}\left|b_{k}\right|^{2}\right) \sum_{|j|=1}^{N}\left(\frac{2 \pi}{T \gamma}\right)^{2} \frac{1}{4 d_{k, j}^{2}-1}=\sum_{k=1}^{N}\left(\gamma_{k}^{4} a_{k, k}+\gamma_{k}^{\prime 4} b_{k, k}\right)\left(\frac{2 \pi}{T \gamma}\right)^{2} \sum_{|j|=1}^{N} \frac{1}{4 d_{k, j}^{2}-1} .
\end{aligned}
$$

We finally have

$A-B \geq \sum_{k=1}^{N}\left(\frac{2 \pi}{T \gamma}\right)^{2}\left(\gamma_{k}^{4} a_{k, k}+\gamma_{k}^{\prime 4} b_{k, k}\right) w_{k}$, with $w_{k}:=\left(\frac{T \gamma}{2 \pi}\right)^{2}-o(1)-\sum_{|j|=1, j \neq k}^{N} \frac{1}{4(k-j)^{2}-1}-\sum_{|j|=1}^{N} \frac{1}{4 d_{k, j}^{2}-1} \succeq 1$,

by using (35), for $M=1 / 2$, and this gives (36).

\section{REFERENCES}

[1] C. Castro and S. Micu, Boundary controllability of a linear semi-discrete 1D wave equation derived from a mixed finite element method. Numer. Math. 102 (2006) 413-462.

[2] R. Glowinski, C.H. Li and J.-L. Lions, A numerical approach to the exact boundary controllability of the wave equation (I), Dirichlet controls: Description of the numerical methods. Japan. J. Appl. Math. 7 (1990) 1-76.

[3] A. Haraux, Séries lacunaires et contrôle semi-interne des vibrations d'une plaque rectangulaire. J. Math. Pures Appl. 68 (1989) 457-465.

[4] L. Ignat, Propiedades cualitativas de esquemas numéricos de aproximción de ecuaciones de difusión y de dispersión. Ph.D. thesis, Universidad Autónoma de Madrid, Spain (2006).

[5] J.A. Infante and E. Zuazua, Boundary observability for the space discretization of the 1D wave equation. ESAIM: M2AN 33 (1999) 407-438.

[6] A.E. Ingham, Some trigonometrical inequalities with applications in the theory of series. Math. Z. 41 (1936) 367-379.

[7] V. Komornik, Exact Controllability and Stabilization. The Multiplier Method. Wiley, Chichester; Masson, Paris (1994).

[8] V. Komornik and P. Loreti, Fourier Series in Control Theory, Springer Monographs in Mathematics. Springer-Verlag, New York (2005).

[9] J.-L. Lions, Contrôlabilité Exacte, Stabilisation et Perturbation de Systèmes Distribués. Tome 1. Contrôlabilité Exacte. Masson, Paris, RMA 8 (1988).

[10] P. Loreti and V. Valente, Partial exact controllability for spherical membranes. SIAM J. Control Optim. 35 (1997) 641-653.

[11] S. Micu, Uniform boundary controllability of a semi-discrete 1D wave equation. Numer. Math. 91 (2002) $723-766$.

[12] S. Micu and E. Zuazua, Boundary controllability of a linear hybrid system arising in the control of noise. SIAM J. Cont. Optim. 35 (1997) 1614-1638. 
[13] A. Münch, Family of implicit and controllable schemes for the 1D wave equation. C. R. Acad. Sci. Paris Sér. I 339 (2004) $733-738$.

[14] M. Negreanu, Numerical methods for the analysis of the propagation, observation and control of waves. Ph.D. thesis, Universidad Complutense Madrid, Spain (2003). Available at http://www.uam.es/proyectosinv/cen/indocumentos.html

[15] M. Negreanu and E. Zuazua, Convergence of a multigrid method for the controllability of a 1D wave equation. C. $R$. Acad. Sci. Paris, Sér. I 338 (2004) 413-418.

[16] M. Negreanu and E. Zuazua, Discrete Ingham inequalities and applications. SIAM J. Numer. Anal. 44 (2006) $412-448$.

[17] E. Zuazua, Propagation, observation, control and numerical approximation of waves approximated by finite difference methods. SIAM Rev. 47 (2005) 197-243.

[18] E. Zuazua, Control and numerical approximation of the wave and heat equations, in Proceedings of the ICM 2006, Vol. III, "Invited Lectures", European Mathematical Society Publishing House, M. Sanz-Solé et al. Eds. (2006) 1389-1417. 